\title{
Pull-Back of Metric Currents and Homological Boundedness of BLD-Elliptic Spaces
}

https://doi.org/10.1515/agms-2019-0011

Received June 10, 2019; accepted October 15, 2019.

Abstract: Using the duality of metric currents and polylipschitz forms, we show that a BLD-mapping $f: X \rightarrow Y$ between oriented cohomology manifolds $X$ and $Y$ induces a pull-back operator $f^{\star}: M_{k, \text { loc }}(Y) \rightarrow M_{k, \text { loc }}(X)$ between the spaces of metric $k$-currents of locally finite mass. For proper maps, the pull-back is a right-inverse (up to multiplicity) of the push-forward $f_{\star}: M_{k, \text { loc }}(X) \rightarrow M_{k, \text { loc }}(Y)$.

As an application we obtain a non-smooth version of the cohomological boundedness theorem of Bonk and Heinonen for locally Lipschitz contractible cohomology $n$-manifolds $X$ admitting a BLD-mapping $\mathbb{R}^{n} \rightarrow X$.

Keywords: Metric currents; BLD-mappings

MSC: 30L10, 49Q15, 30C65

\section{Introduction}

In [4], Ambrosio and Kirchheim extended the Federer-Fleming theory of currents to general metric spaces by viewing currents as multilinear functionals acting on tuples of Lipschitz functions instead of on differential forms; see also Lang [17] for a localized theory on locally compact spaces. Under this formalism, a locally Lipschitz map $f: X \rightarrow Y$ between metric spaces gives rise to a natural push-forward operator $f_{\star}: M_{k}(X) \rightarrow$ $M_{k}(Y)$, given by

$$
f^{\star} T\left(\pi_{0}, \ldots, \pi_{k}\right)=T\left(\pi_{0} \circ f, \ldots, \pi_{k} \circ f\right), \quad\left(\pi_{0}, \ldots, \pi_{k}\right) \in \operatorname{LIP}_{\infty}(X)^{k+1},
$$

between the spaces of finite mass $k$-currents $M_{k}(X)$ and $M_{k}(Y)$, respectively.

In this article we develop a pull-back of metric currents for a special class of Lipschitz maps, called BLD-maps, between locally geodesic oriented cohomology manifolds. A continuous, open and discrete map $f: X \rightarrow Y$ between metric spaces is an $L-B L D$ map, for $L \geq 1$, if it satisfies the bounded length distortion estimate

$$
\frac{1}{L} \ell(y) \leq \ell(f \circ y) \leq L \ell(y)
$$

for each path $y$ in $X$, where $\ell(\cdot)$ is the length of a path. We call a map $f: X \rightarrow Y$ simply a BLD-map if it is $L$-BLD for some $L \geq 1$. Condition (1.2) may be regarded as a locally non-injective variant of the bi-Lipschitz condition. Indeed, every bi-Lipschitz bijection is a BLD-map. Note that, since the spaces we consider are locally geodesic, Condition (1.2) is not vacuous.

Heuristically, the pull-back is a local left inverse of the push-forward; see Theorem 1.1 below. We construct it as an adjoint of a push-forward of polylipschitz forms introduced in [24], which give a pre-dual for metric currents. The construction relies on two key properties of BLD-maps between oriented cohomology manifolds.

Pekka Pankka, University of Helsinki, Helsinki, Finland, E-Mail: pekka.pankka@helsinki.fi *Corresponding Author: Elefterios Soultanis, SISSA, Trieste, Italy and University of Freiburg, Freiburg, Switzerland, E-Mail: elefterios.soultanis@gmail.com 
First, a BLD-map (and more generally a branched cover) $f: X \rightarrow Y$ between oriented cohomology manifolds admits a local index function $i_{f}: X \rightarrow \mathbb{Z}$. For a compactly supported Borel function $g: X \rightarrow \mathbb{R}$, we may define the push-forward $f_{\#} g: Y \rightarrow \mathbb{R}$ of $g$ by $f$ as the function

$$
y \mapsto \sum_{x \in f^{-1}(y)} i_{f}(x) g(x) .
$$

Second, if $X$ and $Y$ are locally geodesic, the lower estimate in (1.2) allows us to obtain a Lipschitz estimate for the push-forward of Lipschitz functions; see Lemma 4.1.

Using these properties we define a push-forward operator

$$
f_{\#}: \Gamma_{c}^{k}(X) \rightarrow \Gamma_{\mathrm{pc}, \mathrm{c}}^{k}(Y)
$$

from the space of polylipschitz forms to the space of partition continuous polylipschitz forms $\Gamma_{\mathrm{pc}, \mathrm{c}}^{\mathrm{k}}(Y)$. We refer to Section 2.2 for polylipschitz forms and Section 4.1 for the details on the push-forward. The pull-back $f^{\star} T$ of a current $T \in M_{k, \text { loc }}(Y)$ of locally finite mass is then obtained by the formula

$$
f^{\star} T\left(\pi_{0}, \ldots, \pi_{k}\right)=\widehat{T}\left(f_{\#}\left[\pi_{0}, \ldots, \pi_{k}\right]\right),
$$

for $\left(\pi_{0}, \ldots, \pi_{k}\right) \in \operatorname{LIP}_{c}(X) \times \operatorname{LIP}_{\infty}(X)^{k}$; see [17] for currents of locally finite mass on locally compact spaces. Here $\widehat{T}$ is the extension of $T$ to the space of piecewise continuous polylipschitz forms; see [24, Theorem 1.3]. More precisely, our main theorem reads as follows. In the statement, $N_{k, \text { loc }}(\cdot)$ is the space of locally normal $k$-currents.

Theorem 1.1. Let $f: X \rightarrow Y$ be an L-BLD map between locally geodesic oriented cohomology manifolds, and let $k \in \mathbb{N}$. Then there is a natural and weakly sequentially continuous linear map

$$
f^{\star}: M_{k, l o c}(Y) \rightarrow M_{k, l o c}(X)
$$

having the following properties:

1. for a -current $T \in M_{k, l o c}(Y)$ and a precompact Borel set $E \subset X$,

$$
f_{\star}\left(\left(f^{\star} T\right)\lfloor E)=T \mid f_{\# \chi_{E}} ;\right.
$$

2. $\partial \circ f^{\star}=f^{\star} \circ \partial: N_{k, \text { loc }}(Y) \rightarrow N_{k-1, \text { loc }}(X)$; and

3. for each $T \in M_{k, \mathrm{loc}}(Y)$,

$$
\frac{1}{L^{k}} f^{\star}\|T\| \leq\left\|f^{\star} T\right\| \leq L^{k} f^{\star}\|T\|,
$$

where $\|\cdot\|$ is the mass measure of a current.

The naturality of the map $f^{\star}: M_{k, \text { loc }}(Y) \rightarrow M_{k, \text { loc }}(X)$ in the statement refers to the typical functorial properties of the pull-back, that is, given BLD-maps $f: X \rightarrow Y$ and $g: Y \rightarrow Z$ between locally geodesic oriented cohomology manifolds, we have the composition rule $f^{\star} \circ g^{\star}=(g \circ f)^{\star}$.

Property (1) in Theorem 1.1 characterizes the pull-back in the following sense.

Theorem 1.2. Let $f: X \rightarrow Y$ be a BLD-map between geodesic oriented cohomology manifolds and $T \in$ $M_{k, l o c}(Y)$. Suppose $S \in M_{k, l o c}(X)$ is such that

$$
f_{\star}\left(S\lfloor E)=T\left\lfloor f_{\# \chi_{E}}\right.\right.
$$

for all precompact Borel sets $E \subset X$. Then

$$
S=f^{\star} T \text {. }
$$

Properties (2) and (3) in Theorem 1.1 immediately imply the stability of normal currents under the pull-back. 
Corollary 1.3. Let $f: X \rightarrow Y$ be a BLD map between locally geodesic oriented cohomology manifolds, and let $k \in \mathbb{N}$. Then the pull-back $f^{\star}: M_{k, \mathrm{loc}}(Y) \rightarrow M_{k, \text { loc }}(X)$ restricts to an operator

$$
f^{\star}: N_{k, \text { loc }}(Y) \rightarrow N_{k, \text { loc }}(X) .
$$

For proper BLD-maps, we may characterize the pull-back as a right inverse of the push-forward as follows. Recall that a map $f: X \rightarrow Y$ is proper if $f^{-1}(K) \subset X$ is compact whenever $K \subset Y$ is compact and that proper maps between oriented cohomology manifolds have a global degree $\operatorname{deg} f \in \mathbb{Z}$.

Corollary 1.4. Let $f: X \rightarrow Y$ be a proper L-BLD-map between locally geodesic, oriented cohomology manifold. Then the pull-back $f^{\star}: M_{k}(Y) \rightarrow M_{k}(X)$ has the following properties:

1. the composition $f_{\star} \circ f^{\star}: M_{k, \text { loc }}(Y) \rightarrow M_{k, \operatorname{loc}}(Y)$ satisfies $f_{\star} \circ f^{\star}=(\operatorname{deg} f)$ id;

2. the pull-back $f^{\star}$ commutes with the boundary, i.e. $\partial f^{\star} T=f^{\star}(\partial T)$ for $T \in M_{k, \text { loc }}(X)$; and

3. for each $T \in M_{k}(X)$,

$$
\frac{1}{L^{k}}(\operatorname{deg} f) M(T) \leq M\left(f^{\star} T\right) \leq L^{k}(\operatorname{deg} f) M(T),
$$

where $M(T)=\|T\|(X)$ is the mass of a finite mass current $T \in M_{k}(X)$.

Moreover, the pull-back operator $f^{\star}$ restricts to a natural operator

$$
f^{\star}: N_{k}(Y) \rightarrow N_{k}(X)
$$

\section{Homological boundedness of BLD-elliptic spaces}

As an application of the pull-back operator for currents, we prove a metric variant of a theorem of BonkHeinonen for BLD-elliptic spaces, i.e. spaces that admit a BLD-map from Euclidean space. This terminology is an adaptation on the notion of quasiregular ellipticity for closed $n$-manifolds admitting a quasiregular map from $\mathbb{R}^{n}$ introduced by Bonk and Heinonen [5], which in turn is an adaptation of ellipticity of a manifold, introduced by Gromov [10].

The theorem of Bonk and Heinonen [5, Theorem 1.1] states that, if $f: \mathbb{R}^{n} \rightarrow M$ is a non-constant $K$ quasiregular map into a closed and oriented Riemannian n-manifold $M$, then there is a constant $C(n, K)$ depending only on $n$ and $K$, such that the de Rham cohomology groups have the dimension bound

$$
\operatorname{dim} H^{k}(M) \leq C(n, K), \quad k=0,1, \ldots, n .
$$

Recently Prywes [25] affirmed the sharp bound $C(n, K)=2^{n}$ conjectured in [5]; see Kangasniemi [15] for a similar result in a dynamical context of uniformly quasiregular mappings.

Theorem 1.5. Suppose $X$ is a compact, geodesic, and locally Lipschitz contractible oriented cohomology manifold which admits an $L-B L D$ map $f: \mathbb{R}^{n} \rightarrow X$. Then there exists a constant $C(n, L)$ depending only on $n$ and $L$ such that

$$
\operatorname{dim} H_{k}(X) \leq C(n, L)
$$

for all $k=0, \ldots, n$.

Here the homology $H_{k}(X)$ is the $k^{\text {th }}$ current homology of $X$, defined using metric currents of Ambrosio and Kirchheim. For locally Lipschitz contractible spaces, the current homology $H_{k}(X)$ agrees with the standard singular homology of $X$. For the definition of oriented cohomology manifolds, we refer to Section 3.2.

Our proof stems from the strategy of Bonk and Heinonen in [5]. Instead of considering pull-backs of $p$ harmonic forms as in [5], we consider pull-backs of metric currents and prove, using compactness and equidistribution, an upper bound for the number of minimal currents spanning the current homology. We find it an interesting question whether it is possible to develop the method of Prywes in this context. 
A comment on the assumptions in Theorem 1.5 is in order. We assume that the target space $X$ is locally Lipschitz contractible, that is, we assume that for every point $x \in X$ and a neighborhood $U$ of $x$ there is a neighborhood $V \subset U$ of $x$ and a Lipschitz map $h: V \times[0,1] \rightarrow U$ for which $h_{0}$ is the inclusion $V \hookrightarrow U$ and $h_{1}$ is a constant map. Clearly, Riemannian manifolds in the theorem of Bonk and Heinonen are locally Lipschitz contractible. In the proof of Theorem 1.5 this assumption yields an a priori finite dimensionality for the current homology $H_{\star}(X)$, which in turn allows us to obtain filling inequality (Proposition 6.3) for normal currents on $X$.

A more commonly used assumption is local linear contractibility, cf. [13]. Local Lipschitz contractibility does not imply local linear contractibility, nor is it implied by it. However, if $X$ satisfies the hypotheses of Theorem 1.5 and is locally linearly contractible, it is a generalized manifold of type A in the terminology of Heinonen and Rickman; see [13, Definition 5.1]. Indeed, local Ahlfors-regularity follows from the work of Heinonen-Rickman [13] and is discussed in Section 5.1 (see Remark 5.1). The remaining condition, local biLipschitz embeddability into some Euclidean space, follows from the work of Almgren, see [3] and De LellisSpadaro [7]. We give the details in Appendix A; see Theorem A.1.

This article is organized as follows. In Sections 2 and 3, we discuss preliminaries on metric currents and polylipschitz forms, and BLD-mappings, respectively. Section 4 is devoted to the pull-back of metric currents under BLD-maps and we prove Theorems 1.1 and 1.2 in this section. In Section 5 we prove equidistribution of the pull-back currents under BLD-maps and in Section 6 we discuss current homology and prove Theorem 1.5. The article is concluded with an appendix on local bilipschitz embeddability of BLD-elliptic spaces into Euclidean spaces.

Acknowledgements We thank Rami Luisto and Stefan Wenger for discussions on the topics of the manuscript.

\section{Metric currents and polylipschitz forms}

In this section, we recall first basic notions from the Ambrosio-Kirchheim theory of metric currents [4] and then briefly discuss the construction of polylipschitz forms introduced in [24].

\subsection{Metric currents}

Let $X$ be a locally compact metric space. A function $f: X \rightarrow \mathbb{R}$ is Lipschitz, if

$$
\operatorname{Lip}(f):=\sup _{x \neq y} \frac{|f(x)-f(y)|}{d(x, y)}<\infty .
$$

We denote by $\operatorname{LIP}_{\infty}(X)$ and $\operatorname{LIP}_{c}(X)$ the vector spaces of bounded Lipschitz functions and Lipschitz functions with compact support, respectively. We equip $\operatorname{LIP}_{\infty}(X)$ and $\operatorname{LIP}_{c}(X)$ with locally convex vector topologies such that

(1) $f_{n} \rightarrow f$ in $\operatorname{LIP} \infty(X)$ if $f_{n} \rightarrow f$ pointwise and $\sup _{n} \operatorname{LIP}\left(f_{n}\right)<\infty$; and

(2) $f_{n} \rightarrow f$ in $\operatorname{LIP}_{c}(X)$ if there is a compact set $K \subset X$ for which $\operatorname{spt}\left(f_{n}\right) \subset K$ for all $n \in \mathbb{N}$, and $f_{n} \rightarrow f$ in $\operatorname{LIP}_{\infty}(X)$.

See [17] for more details. Given $k \geq 0$, let

$$
\mathscr{D}^{k}(X):=\operatorname{LIP}_{c}(X) \times \operatorname{LIP}_{\infty}(X)^{k}
$$

be equipped with the product topology. A $(k+1)$-linear map $T: \mathscr{D}^{k}(X) \rightarrow \mathbb{R}$ is a metric $k$-current on $X$ if

(1) $\lim _{n \rightarrow \infty} T\left(\pi_{n}\right)=T(\pi)$ whenever $\pi_{n} \rightarrow \pi$ in $\mathscr{D}^{k}(X)$, and

(2) $T\left(\pi_{0}, \ldots, \pi_{k}\right)=0$ whenever, for some $j=1, \ldots, k, \pi_{j}$ is constant in a neighbourhood of spt $\pi_{0}$.

The vector space of metric $k$-currents is denoted $\mathscr{D}_{k}(X)$. 


\section{Boundary and restriction}

For $k \geq 1$, the boundary operator $\partial: \mathscr{D}_{k}(X) \rightarrow \mathscr{D}_{k-1}(X)$ is defined by

$$
\partial T\left(\pi_{0}, \ldots, \pi_{k-1}\right):=T\left(\sigma, \pi_{0}, \ldots, \pi_{k-1}\right)
$$

for any $\sigma \in \operatorname{LIP}_{c}(X)$ with $\left.\sigma\right|_{\text {spt } \pi_{0}} \equiv 1$. It follows from the locality condition (2) that the boundary is well defined.

Given $\alpha=\left(\alpha_{0}, \ldots, \alpha_{m}\right) \in \mathscr{D}^{m}(X)$ and $T \in \mathscr{D}_{k}(X)$ with $k \geq m$, we may define the restriction of $T$ by $\alpha$ as the current

$$
T\left\lfloor\alpha \in \mathscr{D}_{k-m}(X), \quad\left(\pi_{0}, \ldots, \pi_{k-m}\right) \mapsto T\left(\alpha_{0} \pi_{0}, \alpha_{1}, \ldots, \alpha_{m}, \pi_{1}, \ldots, \pi_{k-m}\right)\right.
$$

\section{Mass}

A $k$-current $T \in \mathscr{D}_{k}(X)$ is said to have locally finite mass, if there is a Radon measure $\mu$ on $X$ satisfying

$$
\left|T\left(\pi_{0}, \ldots, \pi_{k}\right)\right| \leq \operatorname{Lip}\left(\pi_{1}\right) \cdots \operatorname{Lip}\left(\pi_{k}\right) \int_{X}\left|\pi_{0}\right| \mathrm{d} \mu, \quad\left(\pi_{0}, \ldots, \pi_{k}\right) \in \mathscr{D}^{k}(X)
$$

for every $\left(\pi_{0}, \ldots, \pi_{k}\right) \in \mathscr{D}^{k}(X)$. If $T \in \mathscr{D}_{k}(X)$ has locally finite mass, it admits a mass measure, denoted $\|T\|$, a Radon measure on $X$ that is minimal with respect to satisfying (2.1). If $\|T\|(X)<\infty$, we say that $T$ has finite mass. The space of $k$-currents of locally finite mass is denoted by $M_{k, \text { loc }}(X)$, and the space of $k$-currents of finite mass $M_{k}(X)$.

\section{Normal currents}

A $k$-current $T \in \mathscr{D}_{k}(X)$ is called locally normal, if $T \in M_{k, \text { loc }}(X)$ and $\partial T \in M_{k-1, \text { loc }}(X)$, and normal if $T \in M_{k}(X)$ and $\partial T \in M_{k-1}(X)$. The normal mass $N: N_{k}(X) \rightarrow[0, \infty)$,

$$
T \mapsto\|T\|(X)+\|\partial T\|(X),
$$

is a norm on $N_{k}(X)$ and the normed space $\left(N_{k}(X), N\right)$ is a Banach space; see [17, Proposition 4.2]. For $k=0$, the norm $N$ is the total variation norm.

\section{Flat norm}

Let $E \subset X$ be a Borel set and let $\mathcal{F}_{E}: N_{k, \text { loc }}(X) \rightarrow[0, \infty]$ be the function

$$
T \mapsto \inf \left\{\|T-\partial A\|(E)+\|A\|(E): A \in N_{k+1, \text { loc }}(X)\right\} .
$$

For each non-empty Borel set $E, \mathcal{F}_{E}$ is a seminorm and $\mathcal{F}:=\mathcal{F}_{X}$ is a norm on $N_{k}(X)$, called the flat norm of $N_{k}(X)$.

We recall that, for each $T \in N_{k, \text { loc }}(X)$ and a Borel set $E \subset X$,

$$
\mathcal{F}_{E}(\partial T) \leq \mathcal{F}_{E}(T) \leq\|T\|(E) .
$$

We record standard properties of the flat norm of a restriction of a current as a lemma.

Lemma 2.1. Let $T \in N_{k, \text { loc }}(X)$ and $E \subset X$ a Borel set. Then

$$
\mathcal{F}_{E}\left(T\lfloor\eta) \leq\left(\|\eta\|_{\infty}+\operatorname{Lip} \eta\right) \mathcal{F}_{E}(T)\right.
$$

for all Lipschitz functions $\eta \in \operatorname{LIP}_{\infty}(X)$. Moreover, if $\eta \in \mathrm{LIP}_{\infty}(X)$ satisfies $\left.\eta\right|_{E} \equiv 1$, then

$$
\mathcal{F}_{E}(T)=\mathcal{F}_{E}(T\lfloor\eta) \text {. }
$$


Proof. Let $A \in N_{k, \text { loc }}(X)$ and $B \in N_{k+1, \text { loc }}(X)$ satisfy $T=A+\partial B$. Then, for any $\eta \in \operatorname{LIP}_{c}(X)$ we have

$$
T\lfloor\eta=A\lfloor\eta+(\partial B)\lfloor\eta=A\lfloor\eta+B\lfloor(1, \eta)+\partial(B\lfloor\eta) .
$$

Thus, by (2.1), we have

$$
\begin{aligned}
\mathcal{F}_{E}(T\lfloor\eta) & \leq \| A\lfloor\eta+B\lfloor(1, \eta)\|(E)+\| B\lfloor\eta \|(E) \\
& \leq \| A\lfloor\eta\|(E)+\| B\lfloor(1, \eta)\|(E)+\| B\lfloor\eta \|(E) \\
& \leq\|\eta\|_{\infty}\|A\|(E)+(\operatorname{Lip} \eta)\|B\|(E)+\|\eta\|_{\infty}\|B\|(E) \\
& \leq\left(\|\eta\|_{\infty}+\operatorname{Lip} \eta\right)(\|A\|(E)+\|B\|(E)) .
\end{aligned}
$$

The first claim follows.

For the second claim, note that

$$
\mathcal{F}_{E}(T)=\inf \left\{\|T-\partial\|(E)+\|A\|(E): A \in N_{k+1}(X)\right\},
$$

and that

$$
\| T\lfloor(1-\eta) \|(E)=0
$$

if $\left.\eta\right|_{E} \equiv 1$. For any $A \in N_{k+1}(X)$ we have

$$
\| T\lfloor\eta-\partial A\|(E)-\| T-T\lfloor\eta\|(E) \leq\| T-\partial A\|(E) \leq\| T-T\lfloor\eta\|(E)+\| T\lfloor\eta-\partial A \|(E) .
$$

Thus

$$
\|T-\partial A\|(E)=\| T\lfloor\eta-\partial A \|(E)
$$

and the second claim follows.

The following compactness result for the flat norm provides a crucial tool in the proof of homological boundedness. This result is used for currents in $\mathbb{R}^{n}$ and it is an immediate consequence of [9, Corollary 7.3] and the weak compactness of normal currents [17, Theorem 5.4]. For an analogous compactness result in compact metric spaces, see [8].

Theorem 2.2. Let $A \subset \mathbb{R}^{n}$ be a compact subset and $\lambda \geq 0$. Then the set

$$
N_{k}(A, \lambda)=\left\{T \in N_{k}\left(\mathbb{R}^{n}\right): \text { spt } T \subset A \text { and } N(T) \leq \lambda\right\}
$$

is compact in the flat norm $\mathcal{F}_{A}$, in the sense that every sequence $\left(T_{i}\right)$ in $N_{k}(A, \lambda)$ has a subsequence $\left(T_{i_{k}}\right)$ and $T \in N_{k}(A, \lambda)$ such that

$$
\lim _{k \rightarrow \infty} \mathcal{F}_{A}\left(T_{i_{k}}-T\right)=0
$$

\subsection{Polylipschitz forms}

\section{Polylipschitz functions}

Let $k \in \mathbb{N}$ and $X$ be a metric space. Given functions $f_{0}, \ldots, f_{k}: X \rightarrow \mathbb{R}$, denote by $f_{0} \otimes \cdots \otimes f_{k}: X^{k+1} \rightarrow \mathbb{R}$ the function

$$
\left(x_{0}, \ldots, x_{k}\right) \mapsto f_{0}\left(x_{0}\right) \cdots f_{k}\left(x_{k}\right) .
$$

Note that if each $f_{j}$ is Lipschitz and bounded, then $f_{1} \otimes \cdots \otimes f_{k}$ is Lipschitz and bounded on $X^{k+1}$ (here we endow $X^{k+1}$ with the Euclidean product metric). The norm $L(f)$ on $\operatorname{LIP}_{\infty}(X)$, given by

$$
L(f):=\max \left\{\|f\|_{\infty}, \operatorname{Lip}(f)\right\} \quad \text { for each } f \in \operatorname{LIP}_{\infty}(X),
$$

makes $\operatorname{LIP}_{\infty}(X)$ into a Banach space. 
Consider the algebraic tensor product $\operatorname{LIP}_{\infty}(X)^{\otimes(k+1)}$. The projective tensor norm on $\operatorname{LIP}_{\infty}(X)^{\otimes(k+1)}$ is given by

$$
L_{k}(\pi)=\inf \left\{\sum_{j}^{m} L\left(\pi_{0}^{j}\right) \cdots L\left(\pi_{k}^{j}\right): \pi=\sum_{j}^{m} \pi_{0}^{j} \otimes \cdots \otimes \pi_{k}^{j}\right\}, \quad \pi \in \operatorname{LIP}_{\infty}(X)^{\otimes(k+1)} .
$$

The completion of $\operatorname{LIP}_{\infty}(X)^{\otimes(k+1)}$ with respect to the projective tensor norm is called the (completed) projective tensor product and denoted $\operatorname{LIP}_{\infty}(X)^{\hat{\otimes}_{\pi}(k+1)}$.

The projective tensor product has the following universal property which characterizes it up to isometric isomorphism in the category of Banach spaces: Let $B$ be a Banach space and $A: \operatorname{LIP}_{\infty}(X)^{k+1} \rightarrow B$ a continuous $(k+1)$-linear map. Then there exists a unique continuous linear map $\bar{A}: \operatorname{LIP}_{\infty}(X)^{\hat{\otimes}_{\pi}(k+1)} \rightarrow B$ satisfying

$$
A=\bar{A} \circ \text { J, }
$$

where $\jmath: \operatorname{LIP}_{\infty}(X)^{k+1} \rightarrow \operatorname{LIP}_{\infty}(X)^{\hat{\otimes}_{\pi}(k+1)}$ is the continuous $(k+1)$-linear map $\left(\pi_{0}, \ldots, \pi_{k}\right) \mapsto \pi_{0} \otimes \cdots \otimes \pi_{k}$. In particular, the map

$$
A: \operatorname{LIP}_{\infty}(X)^{k+1} \rightarrow \operatorname{LIP}_{\infty}\left(X^{k+1}\right), \quad\left(\pi_{0}, \ldots, \pi_{k}\right) \mapsto \pi_{0} \otimes \cdots \otimes \pi_{k},
$$

extends to a continuous linear map

$$
\bar{A}: \operatorname{LIP}_{\infty}(X)^{\hat{\otimes}_{\pi}(k+1)} \rightarrow \operatorname{LIP}_{\infty}\left(X^{k+1}\right) .
$$

we identify the projective tensor product with the image of this map in $\operatorname{LIP}_{\infty}\left(X^{k+1}\right)$.

Definition 2.3. Let $X$ be a metric space and $k \in \mathbb{N}$. A function $\pi: X^{k+1} \rightarrow \mathbb{R}$ is a $k$-polylipschitz function on $X$ if there are bounded Lipschitz functions $\pi_{0}^{j}, \ldots \pi_{k}^{j} \in \operatorname{LIP}_{\infty}(X), j=0,1, \ldots$, satisfying

$$
\sum_{j}^{\infty} L\left(\pi_{0}^{j}\right) \cdots L\left(\pi_{k}^{j}\right)<\infty
$$

and

$$
\pi=\sum_{j}^{\infty} \pi_{0}^{j} \otimes \cdots \otimes \pi_{k}^{j}
$$

In other words a polylipschitz function is an element of the completed projective tensor product under the identification explained above.

\section{Polylipschitz forms}

Fix a metric space $X$. Given open sets $U \subset V \subset X$ we denote by

$$
\rho_{U, V}: \operatorname{Poly}^{k}(V) \mapsto \operatorname{Poly}^{k}(U),\left.\quad \pi \mapsto \pi\right|_{U^{k+1}},
$$

the restriction map. The collection

$$
\left\{\operatorname{Poly}^{k}(U), \rho_{U, V}\right\}
$$

ranging over all open sets $U \subset V \subset X$ is known as the (polylipschitz) presheave over $X$. Given $x \in X$ and two polylipschitz functions $\pi \in \operatorname{Poly}^{k}(U), \pi^{\prime} \in \operatorname{Poly}^{k}\left(U^{\prime}\right)$ defined on open neighbourhoods $U$ and $U^{\prime}$ of $x$, respectively, we say that $\pi$ and $\pi^{\prime}$ are equivalent, denoted $\pi \sim \pi^{\prime}$, if there is a neighbourhood $W \subset U \cap U^{\prime}$ such that

$$
\rho_{W, U}(\pi)=\rho_{W, U^{\prime}}\left(\pi^{\prime}\right)
$$

The equivalence class $[\pi]_{x}$ of a polylipschitz $\pi \in \operatorname{Poly}^{k}(U)$ defined on a neighbourhood $U$ of $x$ is called the germ of $\pi$ on $x$. 
The étalé space $\mathcal{P o l y}^{k}(X)$ consists over all such equivalence classes. There is a natural projection map

$$
q: \operatorname{Poly}^{k}(X) \rightarrow X, \quad[\pi]_{x} \mapsto x .
$$

For each $x \in X$, the set

$$
q^{-1}(x)=: \operatorname{Poly}_{x}^{k}(X)
$$

is called the stalk of $\operatorname{Poly}^{k}(X)$ at $x$, and it is a real vector space.

A k-polylipschitz section on $X$ is a section of $\mathcal{P o l y}^{k}(X)$, i.e. a map $\omega: X \rightarrow \mathcal{P o l y}^{k}(X)$ satisfying $q \circ \omega=\mathrm{id}_{X}$. We denote the space of $k$-polylipschitz sections on $X$ by $\mathscr{G}^{k}(X)$. The support of a $k$-polylipschitz section $\omega \in \mathscr{G}^{k}(X)$ is the set

$$
\text { spt } \omega=\operatorname{cl}\{x \in X: \omega(x) \neq 0\} .
$$

The space $\mathcal{P o l}^{k}(X)$ can be equipped with the étalé topology which makes $q$ into a local homeomorphism. See [28, Section 5.6] for the details. Note that $\mathcal{P o l y}^{k}(X)$ is usually a rather pathological space; for example it is rarely Hausdorff. Instead of describing the topology, we describe what continuity of sections means: a section $\omega$ is continuous if there is there is a locally finite open cover $\mathscr{U}$ of $X$ and a collection $\left\{\pi_{U}\right\}_{U \in \mathscr{U}}$, where $\pi_{U} \in \operatorname{Poly}^{k}(U)$, such that $\left[\pi_{U}\right]_{x}=\omega(x)$ for all $U \in \mathscr{U}$ and $x \in U$, and the collection $\left\{\pi_{U}\right\}_{U \in \mathscr{U}}$ satisfies the overlap condition

$$
\rho_{U \cap V, U}\left(\pi_{U}\right)=\rho_{U \cap V, V}\left(\pi_{V}\right)
$$

whenever $U, V \in \mathscr{U}$ and $U \cap V \neq \varnothing$.

Conversely, any collection $\left\{\pi_{U}\right\}_{U \in \mathscr{U}}$ satisfying (2.5) defines a continuous section $\omega$ of $\mathcal{P o l y}^{k}(X)$ by setting

$$
\omega(x)=\left[\pi_{U}\right]_{x}, \quad \text { whenever } U \in \mathscr{U} \text { and } x \in U .
$$

Definition 2.4. Let $X$ be a metric space, and $k \in \mathbb{N}$. A $k$-polylipschitz form on $X$ is a continuous section of $\operatorname{Poly}^{k}(X)$.

The space of $k$-polylipschitz forms on $X$ is denoted by $\Gamma^{k}(X)$, and $\Gamma_{c}^{k}(X)$ denotes the set of polylipschitz forms whose support is compact.

\section{Piecewise continuous polylipschitz forms}

Given any set $B \subset X$, the restriction operators $\rho_{U \cap B, V}: \operatorname{Poly}^{k}(V) \rightarrow \operatorname{Poly}^{k}(U \cap B)$, for $U \subset V \subset X$, form a presheaf homomorphism, giving rise to a restriction homomorphism $\rho_{B}: \mathscr{G}^{k}(X) \rightarrow \mathscr{G}^{k}(B)$, where $B$ is considered as a metric space with the restricted metric from $X$. We denote $\rho_{B}(\omega)=:\left.\omega\right|_{B}$ for $\omega \in \mathscr{G}^{k}(X)$.

Definition 2.5. A k-polylipschitz section $\omega \in \mathscr{G}^{k}(X)$ is called $\mathcal{E}$-continuous, where $\mathcal{E}$ is a countable Borel partition of $X$, if $\left.\omega\right|_{B} \in \Gamma^{k}(B)$ for every $B \in \mathcal{E}$.

A polylipschitz section is partition-continuous if it is $\mathcal{E}$-continuous for some countable Borel partition $\mathcal{E}$ of $X$.

We denote by $\Gamma_{\mathrm{pc}}^{\mathrm{k}}(X)$ the space of partition-continuous polylipschitz sections, and by $\Gamma_{\mathrm{pc}, \mathrm{c}}^{k}(X)$ those elements of $\Gamma_{\mathrm{pc}}^{k}(X)$ which have compact support. Clearly $\Gamma_{c}^{\mathrm{k}}(X) \subset \Gamma_{\mathrm{pc}, \mathrm{c}}^{\mathrm{k}}(X)$.

\section{Exterior derivative and cup-product}

We refer to [24, Section 4.5] for further details. Following the construction of Alexander-Spanier cohomology we introduce the linear map $d=d_{X}^{k}: \operatorname{Poly}^{k}(X) \rightarrow$ Poly $^{k+1}(X)$ by

$$
d \pi\left(x_{0}, \ldots, x_{k+1}\right)=\sum_{j=0}^{k+1}(-1)^{j} \pi\left(x_{0}, \ldots, \hat{x}_{j}, \ldots, x_{k+1}\right)
$$


for $\pi \in \operatorname{Poly}^{k}(X)$ and $x_{0}, \ldots, x_{k+1} \in X$. This map satisfies $d \circ d=0$. The presheaf homomorphism $\left\{d_{U}^{k}\right\}_{U}$ induces homomorphism $d: \mathscr{G}^{k}(X) \rightarrow \mathscr{G}^{k+1}(X)$ that restricts to

$$
\begin{aligned}
& d: \Gamma_{c}^{k}(X) \rightarrow \Gamma_{c}^{k+1}(X) \text { and } \\
& d: \Gamma_{\mathrm{pc}, \mathrm{c}}^{k}(X) \rightarrow \Gamma_{\mathrm{pc}, c}^{k+1}(X) .
\end{aligned}
$$

The cup-product is a bilinear map $\smile: \Gamma_{\mathrm{pc}, \mathrm{c}}^{k}(X) \times \Gamma_{\mathrm{pc}, c}^{m}(X) \rightarrow \Gamma_{\mathrm{pc}, c}^{k+m}(X)$, defined in the same manner starting from the bilinear map

$$
\smile: \operatorname{Poly}^{k}(X) \times \operatorname{Poly}^{m}(X) \rightarrow \operatorname{Poly}^{k+m}(X)
$$

given by

$$
\alpha \smile \beta\left(x_{0}, \ldots, x_{k+m}\right)=\alpha\left(x_{0}, \ldots, x_{k}\right) \beta\left(x_{0}, x_{k+1}, \ldots, x_{k+m}\right)
$$

for $\alpha \in$ Poly $^{k}(X), \beta \in$ Poly $^{m}(X)$ and $x_{0}, \ldots, x_{k+m} \in X$. Note that the cup product restricts to a bilinear map $\smile: \Gamma_{c}^{k}(X) \times \Gamma_{c}^{m}(X) \rightarrow \Gamma_{c}^{k+m}(X)$.

\subsection{Duality of metric currents and polylipschitz forms}

We refer to [24, Sections 4.2, 5.1 and 6] for the notions of convergence of sequences of polylipschitz functions, polylipschitz forms, and partition-continuous polylipschitz forms, respectively. Note that the exterior derivative $d: \Gamma_{\mathrm{pc}, \mathrm{c}}^{k}(X) \rightarrow \Gamma_{\mathrm{pc}, c}^{k+1}(X)$ is sequentially continuous, cf. [24, Proposition 6.8].

Recall the natural embedding

$$
\imath: \mathscr{D}^{k}(X) \hookrightarrow \Gamma_{c}^{k}(X), \quad \imath\left(\pi_{0}, \ldots, \pi_{k}\right)(x)=\left[\pi_{0} \otimes \cdots \otimes \pi_{k}\right]_{x}
$$

for $\left(\pi_{0}, \ldots, \pi_{k}\right) \in \mathscr{D}^{k}(X)$ and $x \in X$. We slightly abuse notation by using the symbol $\imath$ also for the embedding $\mathscr{D}^{k}(X) \hookrightarrow \Gamma_{\mathrm{pc}, \mathrm{c}}^{\mathrm{k}}(X)$.

Theorem 2.6. [24, Theorems 1.1 and 1.3] Let $X$ be a locally compact space, $k \geq 0$, and $T \in M_{k, \text { loc }}(X)$. Then there is a unique sequentially continuous linear functional $\widehat{T}: \Gamma_{\mathrm{pc}, \mathrm{c}}^{\mathrm{k}}(X) \rightarrow \mathbb{R}$ such that

$$
T=\widehat{T} \circ l .
$$

Moreover, if $T \in N_{k+1, \text { loc }}(X)$, then we have

$$
\widehat{\partial T}(\omega)=\widehat{T}(d \omega)
$$

for every $\omega \in \Gamma_{\mathrm{pc}, \mathrm{c}}^{\mathrm{k}}(X)$

Extensions of currents of finite mass also satisfy natural integrability bounds. Given $\pi \in \operatorname{Poly}^{k}(X)$ and $V \subset X$, define a variant of the projective norm $L_{k}(\cdot)$ as follows:

$$
\operatorname{Lip}_{k}(\pi ; V)=\inf \left\{\sum_{j}^{\infty}\left\|\left.\pi_{0}^{j}\right|_{V}\right\|_{\infty} \operatorname{Lip}\left(\left.\pi_{1}^{j}\right|_{V}\right) \cdots \operatorname{Lip}\left(\left.\pi_{k}^{j}\right|_{V}\right): \pi=\sum_{j}^{\infty} \pi_{0}^{j} \otimes \cdots \otimes \pi_{k}^{j}\right\} .
$$

Define the pointwise norm $\|\omega\|_{x}$ of $\omega \in \Gamma^{k}(X)$ at $x \in X$, by

$$
\|\omega\|_{x}:=\inf \left\{\operatorname{Lip}_{k}(\pi ; B(x, r)): r>0\right\}=\lim _{r \rightarrow 0} \operatorname{Lip}_{k}(\pi ; B(x, r))
$$

for any $\pi$ such that $[\pi]_{x}=\omega(x)$. The map $x \mapsto\|\omega\|_{x}$ is easily seen to be upper semicontinuous, cf. [24, Section 6.1].

Proposition 2.7. [24, Theorem 1.2] Suppose $T \in M_{k, \text { loc }}(X)$, and denote by $\|T\|$ the mass measure of $T$. Then

$$
|\widehat{T}(\omega)| \leq \int_{X}\|\omega\|_{x} \mathrm{~d}\|T\|(x), \quad \omega \in \Gamma^{k}(X) .
$$

Remark 2.8. In the forthcoming sections we do not distinguish a metric current $T \in M_{k, \mathrm{loc}}(X)$ from the extension $\widehat{T}$ provided by Theorem 2.6. We will consider metric currents as acting on $\mathscr{D}^{k}(X)$, $\operatorname{Poly}_{c}^{k}(X), \Gamma_{c}^{k}(X)$, or $\Gamma_{\mathrm{pc}, \mathrm{c}}^{\mathrm{k}}(X)$ interchangeably and without mentioning it explicitly. 


\section{Preliminaries on BLD-maps}

\subsection{Branched covers}

A continuous mapping $f: X \rightarrow Y$ between metric spaces is a branched cover if $f$ is discrete and open; recall that the map $f$ is discrete if the pre-image $f^{-1}(y)$ of a point $y \in Y$ is a discrete set, and $f$ is open if the image $f U$ of an open set $U \subset X$ is open. A continuous map $f: X \rightarrow Y$ is proper if the pre-image $f^{-1} E$ of a compact set $E \subset Y$ is compact. In what follows, all mappings between metric spaces are continuous unless otherwise stated.

A pre-compact domain $U \subset X$ is a normal domain of $f: X \rightarrow Y$ if $\partial f U=f \partial U$. Further, if $x \in U$ has the property that $f^{-1} f(x) \cap \bar{U}=\{x\}$, we say that $U$ is a normal neighborhood of $x$ (with respect to $f$ ).

We recall that, given a branched cover $f: X \rightarrow Y$ and a normal domain $U \subset X$ of $f$, the restriction $\left.f\right|_{U}: U \rightarrow f U$ is a proper map; see e.g. Rickman [26] and Väisälä [27].

Let $f: X \rightarrow Y$ be a branched cover between locally compact spaces. For $x \in X$ and $r>0$, we denote by $U_{f}(x, r)$ the connected component of $f^{-1} B(f(x), r)$ containing $x$. When the map $f$ is clear from the context we omit the subscript and write $U(x, r)$ in place of $U_{f}(x, r)$. The following lemma is extensively used throughout the paper. It follows from [18, Lemma 2.1]; see also [26, Lemma I.4.9] and [27, Lemma 5.1.].

Lemma 3.1. Let $f: X \rightarrow Y$ be a branched cover between locally compact spaces $X$ and $Y$. Then the following conditions hold.

(a) For every $x \in X$, there exists a radius $r_{x}>0$, for which $U(x, r)$ is a normal domain of $x$ for every $r<r_{x}$. Furthermore, given a compact set $K \subset X$ and $y \in f(K)$, there exists $r_{y}>0$ so that $U(x, r)$ is a normal neighborhood for $x$, for every $x \in f^{-1}(y) \cap K$ and $r<r_{y}$.

(b) If $U^{\prime} \subset U \subset X$ are normal domains of $x \in X$, then $U \cap f^{-1}\left(f\left(U^{\prime}\right)\right)=U^{\prime}$.

Remark 3.2. It follows that, if $f: X \rightarrow Y$ is a proper branched cover, then, for every $x \in X$, there is a radius $r_{0}>0$ for which

$$
f^{-1} B_{r}(f(x))=\bigcup_{x^{\prime} \in f^{-1}(f(x))} U\left(x^{\prime}, r\right),
$$

where $U\left(x^{\prime}, r\right)$ is a normal neighborhood of $x^{\prime} \in f^{-1}(f(x))$, for each $x^{\prime} \in f^{-1}(f(x))$.

\subsection{Oriented cohomology manifolds}

Following [13] we say that a separable and locally compact space $X$ is an oriented cohomology $n$-manifold if

(a) $X$ has finite covering dimension,

(b) $H_{c}^{k}(U ; \mathbb{Z})=0$ for each open set $U \subset X$ for $k \geq n+1$,

(c) $H_{c}^{n}(X ; \mathbb{Z}) \simeq \mathbb{Z}$, and

(d) each point $x$ and its neighborhood $U$ contains a neighborhood $V \subset U$ of $x$ for which

$$
H_{c}^{k}(V ; \mathbb{Z})= \begin{cases}0 & , k=n-1 \\ \mathbb{Z} & , k=n\end{cases}
$$

and the standard homomorphism

$$
H_{c}^{n}(W ; \mathbb{Z}) \rightarrow H_{c}^{n}(V ; \mathbb{Z})
$$

is a surjection for any neighborhood $W$ of $x$ contained in $V$.

The notation $H_{c}^{*}(-; \mathbb{Z})$ above refers to the compactly supported Alexander-Spanier cohomology with integer coefficients. We refer to [13, Definition 1.1] and the ensuing discussion for more details. Here we only mention that a more widely used notion of cohomology manifolds requires all local cohomology groups of dimension $0<k<n$ to vanish, see e.g. [6, Definition 6.17]. 


\subsection{Global and local degree}

Let $X$ and $Y$ be oriented cohomology manifolds of the same dimension $n \in \mathbb{N}$ and fix orientations $c_{X}$ and $c_{Y}$ of $X$ and $Y$, i.e. generators $c_{X}$ and $c_{Y}$ of $H_{c}^{n}(X ; \mathbb{Z})$ and $H_{c}^{n}(Y ; \mathbb{Z})$, respectively. For open sets $U \subset X$ and $V \subset Y$ we have local orientations given by $c_{U}=\iota_{U X}^{\star} c_{X}$ and $c_{V}=\iota_{V Y}^{\star} c_{Y}$, where $\iota_{U X}: U \hookrightarrow X$ and $\iota_{V Y}: V \hookrightarrow Y$ are inclusions. As described in [13, 26, 27], continuous maps $X \rightarrow Y$ admit a local degree in the following sense. Here we follow the presentation in [13].

Given a precompact domain $U \subset X$, the local degree $\mu_{f}(U, y) \in \mathbb{Z}$ with respect to a point $y \in Y \backslash f(\partial U)$ and domain $U$ is

1. 0 if $y \notin \operatorname{int} f(U)$, and otherwise

2. the unique integer $\lambda \in \mathbb{Z}$ for which the pull-back homomorphism

$$
\left(\left.f\right|_{U}\right)^{\star}: H_{c}^{n}(V) \rightarrow H_{c}^{n}(U) \text { satisfies } f^{\star}\left[c_{V}\right]=\lambda\left[c_{U}\right],
$$

where $V$ is the component of int $f(U) \backslash f(\partial U)$ containing $y$; note that $y \in \operatorname{int} f(U)$ in this case.

Then $\mu_{f}(U, y)$ is constant in each component of int $f(U) \backslash f(\partial U)$.

If $f: X \rightarrow Y$ is a proper map, it admits a global degree $\operatorname{deg} f$, which is the unique integer $\lambda \in \mathbb{Z}$ for which the pull-back $f^{\star}: H_{c}^{n}(Y) \rightarrow H_{c}^{n}(X)$ in cohomology satisfies

$$
f^{\star} c_{Y}=\lambda c_{X} .
$$

A standard property of the local degree is that, for precompact domains $V \subset U$ and a point $y \in Y$ satisfying $y \notin f(\partial V) \cup f(\partial U)$ and $f^{-1}(y) \cap U \subset V$, we have

$$
\mu_{f}(V, y)=\mu_{f}(U, y) .
$$

This immediately yields a summation formula

$$
\mu_{f}(U, y)=\sum_{i=1}^{N} \mu_{f}\left(U_{i}, y\right)
$$

for pairwise disjoint domains $U_{1}, \ldots, U_{N}$ contained in $U$ and satisfying

$$
y \notin f\left(\partial U_{1}\right) \cup \cdots \cup f\left(\partial U_{N}\right) \text { and } f^{-1}(y) \cap U \subset U_{1} \cup \cdots \cup U_{N} .
$$

As a consequence we obtain that, for a branched cover $f: X \rightarrow Y$, the local degree function $i_{f}: X \rightarrow \mathbb{Z}$, defined by

$$
x \mapsto \mu_{f}(U, f(x)),
$$

where $U$ is any normal neighborhood of $x$, is well-defined. For branched covers, we may express the summation formula (3.1) in terms of the local index. Indeed, let $f: X \rightarrow Y$ be a branched cover between oriented cohomology manifolds of the same dimension and suppose $U \subset X$ is a normal domain for $f$. Then

$$
\mu_{f}(U, y)=\sum_{x \in f^{-1}(y) \cap U} i_{f}(x)
$$

for $y \in Y \backslash f(\partial U)$. If $f$ is a proper branched cover then

$$
\operatorname{deg} f=\sum_{x \in f^{-1}(y)} i_{f}(x)
$$

for any $y \in f(X)$; see [26] and [13].

The local index satisfies a chain rule analogous to the chain rule for derivatives. More precisely, given branched covers $f: X \rightarrow Y$ and $g: Y \rightarrow Z$ between oriented cohomology manifolds, we have that

$$
i_{g \circ f}(x)=i_{g}(f(x)) i_{f}(x)
$$


for all $x \in X$.

A branched cover $f: X \rightarrow Y$ is sense preserving (sense reversing) if $\mu_{f}(D, y)>0\left(\mu_{f}(D, y)<0\right)$ for all precompact domains $D \subset X$ and $y \in f(D) \backslash f(\partial D)$. It is known that branched cover between oriented cohomology manifolds is either sense preserving or sense reversing [27]. Thus we may always choose the orientations $c_{X}$ and $c_{Y}$ of $X$ and $Y$, respectively, so that a given branched cover $f$ is sense preserving. In particular we may assume $i_{f} \geq 1$ everywhere.

\section{Branch set}

Local homeomorphisms are always branched covers. However the converse fails, that is, a branched cover $f: X \rightarrow Y$ between oriented cohomology manifolds need not be a local homeomorphism. We define the set $B_{f}$ to be the set of points $x \in X$ for which $f$ is not a local homeomorphism at $x$. The branch set is easily seen to be a closed set.

It is known that the branch set $B_{f}$ as well as its image $f B_{f}$ of a branched cover between oriented cohomology $n$-manifolds has topological dimension at most $n-2$; see [27]. In particular $B_{f}$ and $f B_{f}$ do not locally separate $X$ and $Y$, respectively, that is, $U \backslash B_{f}$ (resp. $V \backslash f B_{f}$ ) is path connected for every open $U \subset X$ (resp. $V \subset Y$ ); see also [13, 3.1].

An orientation preserving proper branched cover $f: X \rightarrow Y$ is $(\operatorname{deg} f)$-to-one in the sense that, for any $y \in Y \backslash f B_{f}$, the preimage $f^{-1}(y)$ contains exactly $\operatorname{deg} f$ points.

\subsection{BLD-maps and path-lifting}

A BLD-map $f: X \rightarrow Y$ between metric spaces $X$ and $Y$ is a branched cover satisfying the bounded length distortion inequality (1.2) for some $L \geq 1$. BLD-maps first appeared in [19] as a subclass of quasiregular maps between Euclidean spaces, and in [13] in the present metric context. We refer to [18] for alternative characterizations of BLD-maps between metric spaces.

A path-lifting yields a bijection between preimages of points not in the image of the branch set of the map. In what follows, we use the following version of [18, Lemma 4.4]. We omit the details.

Lemma 3.3. Let $f: X \rightarrow Y$ be an L-BLD map between two oriented cohomology manifolds. Suppose there exists a geodesic joining $p, q \notin f B_{f}$. Let $K \subset X$ is a compact set. Then there is a bijection $\psi: f^{-1}(p) \rightarrow f^{-1}(q)$ satisfying

$$
d(p, q) / L \leq d(x, \psi(x)) \leq L d(p, q)
$$

for every $x \in f^{-1}(p) \cap K$.

\section{The pull-back of metric currents by BLD-maps}

Given a branched cover $f: X \rightarrow Y$ and set $E \subset X$ we say that a ball $B_{r}(y) \subset Y$ is a spread neighborhood (of $y \in Y$ ) with respect to $E$ if $U_{f}(x, r)$ is a normal neighborhood of $x$ for each $x \in f^{-1}(y) \cap E$. We say that $B_{r}(y)$ is a spread neighborhood if it is a spread neighborhood with respect to $X$.

Recall that, by Lemma 3.1, for a compact set $K \subset X$, sufficiently small balls $B_{r}(y)$, for $y \in Y$ and $r>0$, are spread neighborhoods with respect to $K$. By Remark 3.2, sufficiently small balls $B_{r}(y)$, for $y \in Y$ and $r>0$, are spread neighborhoods for proper BLD-maps.

We say that a metric space $X$ is locally geodesic if any point $x \in X$ has a neighborhood $U \subset X$ with the property that, for any two points $p, q \in U$, there is a geodesic joining them, i.e. a curve $y:[0, d(p, q)] \rightarrow X$ satisfying

$$
d(p, q)=\ell(y)
$$


We call such neighborhoods geodesic neighborhoods. Note, however, that the geodesic $y$ is not required to lie inside the neighborhood $U$. We also say that a ball $B_{r}(y) \subset Y$ is a geodesic spread neighborhood with respect to a set $E \subset X$ if it is both a geodesic neighborhood, and a spread neighborhood with respect to $E$. Similarly, a geodesic spread neighborhood is a spread neighborhood that is also a geodesic neighborhood.

We use the notation $y: x \curvearrowright y$ to denote a curve $y:[a, b] \rightarrow X$ joining two points $x, y \in X$.

In what follows, we consider only locally geodesic oriented cohomology manifolds.

\section{Push-forward of functions by BLD-maps}

Recall that the push-forward of a compactly supported Borel function $g: X \rightarrow \mathbb{R}$ by a BLD-map $f: X \rightarrow Y$ is the function $f_{\sharp} g: Y \rightarrow \mathbb{R}$,

$$
y \mapsto \sum_{x \in f^{-1}(x)} i_{f}(x) g(x) .
$$

It is not difficult to see that the push-forward $f_{\sharp} g$ is a Borel function.

Lemma 4.1. Let $f: X \rightarrow Y$ be a proper L-BLD map. Given a Lipschitz function $\eta \in \operatorname{LIP}_{c}(X)$, the push-forward $f_{\#} \eta: Y \rightarrow \mathbb{R}$ is locally Lipschitz and satisfies the bound

$$
\operatorname{Lip} f_{\#} \eta(y) \leq L(\operatorname{deg} f) \operatorname{Lip}(\eta)
$$

for each $y \in Y$. Furthermore, $f_{\#} \eta$ satisfies the estimate

$$
\left\|f_{\#} \eta\right\|_{\infty} \leq(\operatorname{deg} f)\|\eta\|_{\infty} .
$$

Proof. The second estimate follows by a direct computation. Indeed, for any $p \in Y$, we have

$$
\left|f_{\#} \eta(p)\right| \leq \sum_{x \in f^{-1}(p)} i_{f}(x)\|\eta\|_{\infty}=(\operatorname{deg} f)\|\eta\|_{\infty},
$$

by the summation formula (3.2) for the local index.

We now prove the first estimate. Let $p \in Y$ and take a geodesic spread neighborhood $B_{r}(p)$ of $p$. The preimage

$$
f^{-1} B_{r}(p)=\bigcup_{x \in f^{-1}(p)} U_{X}
$$

is a mutually disjoint union of normal neighborhoods $U_{x}$ of preimage points $x$. For any $q \in B_{r}(p)$ we have

$$
f_{\#} \eta(q)=\sum_{y \in U \cap f^{-1}(q)} i_{f}(y) \eta(y)=\sum_{x \in f^{-1}(p)} \sum_{y \in f^{-1}(q) \cap U_{x}} i_{f}(y) \eta(y)
$$

and further

$$
\left|f_{\#} \eta(p)-f_{\#} \eta(q)\right| \leq \sum_{x \in f^{-1}(p)}\left|i_{f}(x) \eta(x)-\sum_{y \in f^{-1}(q) \cap U_{x}} i_{f}(y) \eta(y)\right| .
$$

By substituting the local summation formula (3.2) into (4.1) we have the estimate

$$
\begin{aligned}
\left|f_{\#} \eta(p)-f_{\#} \eta(q)\right| & \leq \sum_{x \in f^{-1}(p)}\left|\sum_{y \in f^{-1}(p) \cap U_{x}} i_{f}(y)(\eta(x)-\eta(y))\right| \\
& \leq \sum_{x \in f^{-1}(p)} \sum_{y \in f^{-1}(p) \cap U_{x}} i_{f}(y)|\eta(x)-\eta(y)| \\
& \leq \operatorname{Lip}(\eta) \sum_{x \in f^{-1}(p)} \sum_{y \in f^{-1}(p) \cap U_{x}} i_{f}(y) d(x, y) .
\end{aligned}
$$


For each $y \in U_{x}$, we have

$$
d(x, y) \leq \ell\left(y^{\prime}\right) \leq L \ell(y)=L d(p, q),
$$

where $y^{\prime}: x \curvearrowright y$ is a lift of a geodesic $y: p \curvearrowright q$. Thus

$$
\begin{aligned}
\left|f_{\#} \eta(p)-f_{\#} \eta(q)\right| & \leq \operatorname{Lip}(\eta) \operatorname{Ld}(p, q) \sum_{x \in f^{-1}(p)} \sum_{y \in f^{-1}(p) \cap U_{x}} i_{f}(y) \\
& =\operatorname{Lr} \operatorname{Lip}(\eta) \sum_{x \in f^{-1}(p)} i_{f}(x)=L d(p, q) \operatorname{Lip}(\eta) \operatorname{deg} f .
\end{aligned}
$$

It follows that $\operatorname{lip} f_{\#} \eta(p) \leq L \operatorname{Lip}(\eta)$ for every $p \in Y$. Suppose that $y \in Y$ and $B_{r}(y)$ is a spread neighborhood. Then, for any $p, q \in B_{r}(y)$, choosing a geodesic $y$ connecting them, we have

$$
\left|f_{\#} \eta(p)-f_{\#} \eta(q)\right| \leq \int_{0}^{1} \operatorname{Lip} f_{\#} \eta(y(t))\left|\dot{y}_{t}\right| \mathrm{d} t \leq L d(p, q)(\operatorname{deg} f) \operatorname{Lip}(\eta) .
$$

This proves that $f_{\#} \eta$ is locally Lipschitz and satisfies the first estimate in the claim.

The following lemma shows that the push-forward is natural with respect to composition.

Lemma 4.2. Let $f: X \rightarrow Y$ and $g: Y \rightarrow Z$ be proper BLD-maps between locally geodesic oriented cohomology manifolds. Given a Borel function $h: X \rightarrow \mathbb{R}$ we have

$$
g_{\#}\left(f_{\#} h\right)(z)=(g \circ f)_{\#} h(z)
$$

for every $z \in Z$.

Proof. Let $z \in Z$. Then

$$
(g \circ f)^{-1}(z)=\bigcup_{y \in g^{-1}(z)} f^{-1}(y)
$$

and, by (3.4), $i_{g \circ f}(x)=i_{g}(f(x)) i_{f}(x)$ for any $x \in(g \circ f)^{-1}(z)$.

Thus

$$
\begin{aligned}
g_{\#}\left(f_{\#} h\right)(z) & =\sum_{y \in g^{-1}(z)} i_{g}(y) f_{\#} h(y)=\sum_{y \in g^{-1}(z)} \sum_{x \in f^{-1}(y)} i_{g}(y) i_{f}(x) h(x) \\
& =\sum_{y \in g^{-1}(z)} \sum_{x \in f^{-1}(y)} i_{g}(f(x)) i_{f}(x) h(x)=\sum_{x \in(g \circ f)^{-1}(z)} i_{g \circ f}(x) h(x) \\
& =(g \circ f)_{\#} h(z)
\end{aligned}
$$

for every $z \in Z$.

\section{Push-forward of polylipschitz functions by BLD-maps}

To simplify notation, we denote by $\bar{x}=\left(x_{0}, \ldots, x_{k}\right)$ a $(k+1)$-tuple of points in $X^{k+1}$. If $g: X \rightarrow \mathbb{R}$ is a function we define $\bar{g}: X^{k+1} \rightarrow \mathbb{R}$ by

$$
\bar{x} \mapsto g\left(x_{0}\right) \cdots g\left(x_{k}\right) .
$$

For example, for the local index $i_{f}: X \rightarrow \mathbb{Z}$ of a BLD-map $f: X \rightarrow Y$, we denote

$$
\overline{i_{f}}(\bar{x})=i_{f}\left(x_{0}\right) i_{f}\left(x_{1}\right) \cdots i_{f}\left(x_{k}\right)
$$

for $\bar{x}=\left(x_{0}, \ldots, x_{k}\right) \in X^{k+1}$.

Let $f: X \rightarrow Y$ be a BLD-map between locally geodesic, oriented cohomology manifolds. Let $U \subset X$ be a normal domain for $f$. Given a normal domain $U \subset X$ for $f$, consider the continuous $(k+1)$-linear linear map

$$
A_{f}^{U}: \operatorname{LIP}_{\infty}(U)^{k+1} \rightarrow \operatorname{Poly}^{k}(f U), \quad\left(\pi_{0}, \ldots, \pi_{k}\right) \mapsto\left(\left(\left.f\right|_{U}\right)_{\#} \pi_{0}\right) \otimes \cdots \otimes\left(\left(\left.f\right|_{U}\right)_{\#} \pi_{k}\right) .
$$


Definition 4.3. Let $f: X \rightarrow Y$ be a BLD-map between locally geodesic, oriented cohomology manifolds, and let $U \subset X$ be a normal domain for $f$. The push-forward

$$
f_{U \#}: \operatorname{Poly}^{k}(U) \rightarrow \operatorname{Poly}^{k}(f U)
$$

is the unique continuous linear extension of $A_{f}^{U}$ for which (2.2) holds.

By the linearity and the sequential continuity of $f_{U \#}$ we have that, if $\pi \in \operatorname{Poly}^{k}(U)$ and $\left(\pi_{0}^{j}, \ldots, \pi_{k}^{j}\right) \in \operatorname{Rep}(\pi)$, then

$$
f_{U \#} \pi=\sum_{j}^{\infty}\left(\left(\left.f\right|_{U}\right)_{\#} \pi_{0}^{j}\right) \otimes \cdots \otimes\left(\left(\left.f\right|_{U}\right)_{\#} \pi_{k}^{j}\right) .
$$

Denote $\operatorname{deg}\left(\left.f\right|_{U}\right)=\mu_{f}(U)$ and let $L$ be the BLD-constant of $f$. Lemma 4.1 and (4.2) immediately yield the estimates

$$
L_{k}\left(f_{U \#} \pi ; f U\right) \leq L^{k+1} \mu_{f}(U)^{k+1} \sum_{j}^{\infty} L\left(\left.\pi_{0}^{j}\right|_{U}\right) \cdots L\left(\left.\pi_{k}^{j}\right|_{U}\right)
$$

and

$$
\operatorname{Lip}_{k}\left(f_{U \#} \pi: f U\right) \leq L^{k} \mu_{f}(U)^{k+1} \sum_{j}^{\infty}\left\|\left.\pi_{0}^{j}\right|_{U}\right\|_{\infty} \operatorname{Lip}\left(\left.\pi_{1}^{j}\right|_{U}\right) \cdots \operatorname{Lip}\left(\left.\pi_{k}^{j}\right|_{U}\right)
$$

for every $\pi \in \operatorname{Poly}^{k}(U)$ and $\left(\pi_{0}^{j}, \ldots, \pi_{k}^{j}\right) \in \operatorname{Rep}(\pi)$. We obtain the following corollary.

Corollary 4.4. Let $f: X \rightarrow Y$ an L-BLD-map between locally geodesic, oriented cohomology manifolds, and let $U \subset X$ be a normal domain for $f$. Then

$$
L_{k}\left(f_{U \#} \pi ; f U\right) \leq L^{k+1} \mu_{f}(U)^{k+1} L_{k}(\pi ; U)
$$

and

$$
\operatorname{Lip}_{k}\left(f_{U \#} \pi ; f U\right) \leq L^{k} \mu_{f}(U)^{k+1} \operatorname{Lip}_{k}(\pi ; U) .
$$

Lemma 4.5. (Restriction principle) Let $f: X \rightarrow Y$ be a BLD-map between locally geodesic oriented cohomology manifolds, and $x \in X$. Then, if $U^{\prime} \subset U \subset X$ are normal domains for $x$ and if $\pi \in \operatorname{Poly}^{k}(U)$, we have

$$
f_{U \#} \pi(\bar{z})=f_{U^{\prime} \#} \pi(\bar{z})
$$

for all $\bar{z} \in f\left(U^{\prime}\right)^{k+1}$.

Proof. By Lemma 3.1 (b), we have that $U \cap f^{-1}\left(f\left(U^{\prime}\right)\right)=U^{\prime}$. Thus, for all $z \in f\left(U^{\prime}\right)$,

$$
U^{\prime} \cap f^{-1}(z)=U \cap f^{-1}(z) .
$$

The claim follows from this immediately.

For the next three lemmas, we assume that $f: X \rightarrow Y$ is an $L$-BLD map between geodesic, oriented cohomology manifolds, $U \subset X$ is a normal domain for $f$, and that $k \geq 0$ is a fixed integer. We show that the push-forward commutes with the cup product and the exterior derivative.

Lemma 4.6. Given $\pi \in \operatorname{Poly}^{k}(U)$ and $\sigma \in \operatorname{Poly}^{m}(f U)$ we have

$$
f_{U \#}\left(\pi \smile f^{\#} \sigma\right)=\mu_{f}(U)^{m}\left(f_{U \#} \pi\right) \smile \sigma .
$$

Proof. We observe first that, given functions $g, h: U \rightarrow \mathbb{R}$ and $p \in f U$, we have

$$
\left(\left.f\right|_{U}\right)_{\#}(g(h \circ f))(p)=\sum_{x \in U \cap f^{-1}(p)} i_{f}(x) g(x) h(f(x))=h(p)\left(\left.f\right|_{U}\right)_{\#} g(p) .
$$


Now let $\pi=\pi_{0} \otimes \cdots \otimes \pi_{k} \in \operatorname{Poly}^{k}(U)$ and $\sigma=\sigma_{0} \otimes \cdots \otimes \sigma_{m} \in \operatorname{Poly}^{m}(f U)$ be polylipschitz functions. Then

$$
\begin{aligned}
& f_{U \#}\left(\pi \smile f^{\#} \sigma\right)=f_{U \#}\left(\pi_{0}\left(\sigma_{0} \circ f\right) \otimes \pi_{1} \otimes \cdots \otimes \pi_{k} \otimes\left(\sigma_{1} \circ f\right) \otimes \cdots \otimes\left(\sigma_{k} \circ f\right)\right) \\
& =f_{U \#}\left(\pi_{0}\left(\sigma_{0} \circ f\right)\right) \otimes f_{U \#}\left(\pi_{1}\right) \otimes \cdots \otimes f_{U \#}\left(\pi_{k}\right) f_{U \#}\left(\sigma_{1} \circ f\right) \otimes \cdots \otimes f_{U \#}\left(\sigma_{k} \circ f\right) \\
& =\left(\sigma_{0} f_{U \#} \pi_{0}\right) \otimes f_{U \#} \pi_{1} \otimes \cdots \otimes f_{U \#} \pi_{k} \otimes\left(\mu_{f}(U) \sigma_{1}\right) \otimes \cdots \otimes\left(\mu_{f}(U) \sigma_{m}\right) \\
& =\mu_{f}(U)^{m}\left(f_{U \#} \pi\right) \smile \sigma .
\end{aligned}
$$

Since the cup product is bi-linear and the pull-back is linear we have, by (4.2), that the claim holds for all $\pi \in \operatorname{Poly}^{k}(U)$ and $\sigma \in \operatorname{Poly}^{m}(V)$.

Lemma 4.7. For each $\pi \in \operatorname{Poly}^{k}(U)$, we have

$$
f_{U \#}(d \pi)=\mu_{f}(U) d f_{U \#} \pi .
$$

Proof. As before, it suffices to consider the case $\pi=\pi_{0} \otimes \cdots \otimes \pi_{k} \in \operatorname{Poly}^{k}(U)$. Then

$$
\begin{aligned}
f_{U \#}(d \pi) & =\sum_{l=0}^{k+1}(-1)^{l} f_{U \#}\left(\pi_{0} \otimes \cdots \otimes \pi_{l-1} \otimes 1 \otimes \pi_{l} \otimes \cdots \otimes \pi_{k}\right) \\
& =\sum_{l=0}^{k+1}(-1)^{l} f_{U \#}\left(\pi_{0} \otimes \cdots \otimes \pi_{l-1}\right) \otimes \mu_{f}(U) \otimes f_{U \#}\left(\pi_{l} \otimes \cdots \otimes \pi_{k}\right) \\
& =\mu_{f}(U) d f_{U \#} \pi .
\end{aligned}
$$

The following lemma shows that the push-forward is sequentially continuous.

Lemma 4.8. Suppose $\pi^{n} \rightarrow \pi$ in $\operatorname{Poly}^{k}(U)$. Then $f_{U \#} \pi^{n} \rightarrow f_{U \#} \pi$ in $\operatorname{Poly}^{k}(V)$.

Proof. Since $\pi^{n} \rightarrow \pi$ in $\operatorname{Poly}^{k}(U)$ there is, for every $n \in \mathbb{N}$, a representation

$$
\pi^{n}-\pi=\sum_{j}^{\infty} \pi_{0}^{j, n} \otimes \cdots \otimes \pi_{k}^{j, n}
$$

of $\pi^{n}-\pi$ satisfying

$$
\sup _{n \in \mathbb{N}} \sum_{j}^{\infty} L\left(\left.\pi_{0}^{j, n}\right|_{U}\right) \cdots L\left(\left.\pi_{k}^{j, n}\right|_{U}\right)<\infty \text { and } \lim _{n \rightarrow \infty} \sum_{j}^{\infty}\left\|\left.\pi_{0}^{j, n}\right|_{U}\right\|_{\infty} \cdots\left\|\left.\pi_{k}^{j, n}\right|_{U}\right\|_{\infty}=0 .
$$

Since

$$
f_{U \#} \pi_{n}-f_{U \#}=f_{U \#}\left(\pi^{n}-\pi\right)=\sum_{j}\left(f_{U \#} \pi_{0}^{j, n}\right) \otimes \cdots \otimes\left(f_{U \#} \pi_{k}^{j, n}\right),
$$

we have, by the estimates in Lemma 4.1, that

$$
\begin{aligned}
& \sup _{n \in \mathbb{N}} \sum_{j}^{\infty} L\left(\left.f_{U \#} \pi_{0}^{j, n}\right|_{V}\right) \cdots L\left(\left.f_{U \#} \pi_{k}^{j, n}\right|_{V}\right) \\
& \quad \leq L^{k+1} \mu_{f}(U)^{k+1} \sup _{n \in \mathbb{N}} \sum_{j}^{\infty} L\left(\left.\pi_{0}^{j, n}\right|_{U}\right) \cdots L\left(\left.\pi_{k}^{j, n}\right|_{U}\right)<\infty
\end{aligned}
$$

and

$$
\sum_{j}^{\infty}\left\|\left.f_{U \#} \pi_{0}^{j, n}\right|_{V}\right\|_{\infty} \cdots\left\|\left.f_{U \#} \pi_{k}^{j, n}\right|_{V}\right\|_{\infty} \leq \mu_{f}(U)^{k+1} \sum_{j}^{\infty}\left\|\left.\pi_{0}^{j, n}\right|_{U}\right\|_{\infty} \cdots\left\|\left.\pi_{k}^{j, n}\right|_{U}\right\|_{\infty} \rightarrow 0
$$

as $n \rightarrow \infty$. Thus $f_{U \#} \pi^{n} \rightarrow f_{U \#} \pi$ in $\operatorname{Poly}^{k}(V)$. 
Finally, we show that the push-forward is natural in the sense that the composition of push-forwards is the push-forward of compositions

Lemma 4.9. Let $f: X \rightarrow Y$ and $g: Y \rightarrow Z$ be BLD-maps between locally geodesic oriented cohomology manifolds. Let $U \subset X$ be a normal domain for $f$ and $V \subset f(U)$ a normal domain for $g$. Set $W=g(V)$ and $U^{\prime} \subset f^{-1}(V) \cap U$ a component of $f^{-1}(V) \cap U$. Then

$$
g_{V \#} \circ f_{U^{\prime} \#} \pi=(g \circ f)_{U^{\prime} \#} \pi
$$

for every $\pi \in \operatorname{Poly}^{k}\left(U^{\prime}\right)$.

Proof. We observe first that $U^{\prime}$ is a normal domain for $f$. Let $\pi=\pi_{0} \otimes \cdots \otimes \pi_{k} \in$ Poly $^{k}\left(U^{\prime}\right)$. By Lemma 4.2, we have

$$
\left(\left.g\right|_{V)_{\#}}\left(\left.f\right|_{U^{\prime}}\right)_{\#} \pi_{j}=\left(\left.(g \circ f)\right|_{U^{\prime}}\right)_{\#} \pi_{j}\right.
$$

on $W$, for each $j=0, \ldots, k$. Thus

$$
\begin{aligned}
\left(g_{V \#} \circ f_{U^{\prime} \#}\right) \pi & =g_{V \#}\left(\left(\left.f\right|_{U^{\prime}}\right)_{\#} \pi_{0} \otimes \cdots \otimes\left(\left.f\right|_{U^{\prime}}\right)_{\#} \pi_{k}\right) \\
& =\left(\left(\left.g\right|_{V}\right)_{\#}\left(\left.f\right|_{U^{\prime}}\right)_{\#} \pi_{1} \otimes \cdots \otimes\left(\left(\left.g\right|_{V}\right)_{\#}\left(\left.f\right|_{U^{\prime}}\right)_{\#} \pi_{k}\right)\right. \\
& =\left(\left.(g \circ f)\right|_{U^{\prime}}\right)_{\#} \pi_{0} \otimes \cdots \otimes\left(\left.(g \circ f)\right|_{U^{\prime}}\right)_{\#} \pi_{k}=(g \circ f)_{U^{\prime} \#} \pi .
\end{aligned}
$$

By (4.2), equality holds for all $\pi \in \operatorname{Poly}^{k}\left(U^{\prime}\right)$.

\subsection{Push-forward of polylipschitz forms}

Let $f: X \rightarrow Y$ be a BLD-map between locally geodesic oriented cohomology manifolds $X$ and $Y$. We show that the push-forwards $f_{U \#}: \operatorname{Poly}^{k}(U) \rightarrow \operatorname{Poly}^{k}(f(U))$, where $U \subset X$ is a normal domain for $f$, induce a map $\operatorname{Poly}^{k}(X) \rightarrow \operatorname{Poly}^{k}(Y)$.

Lemma 4.10. Let $f: X \rightarrow Y$ be a BLD-map between locally geodesic oriented cohomology manifolds, and let $x \in X$. Let $U$ and $U^{\prime}$ be normal neighborhoods of $x$, and let $\pi \in \operatorname{Poly}^{k}(U), \pi^{\prime} \in \operatorname{Poly}^{k}\left(U^{\prime}\right)$ be polylipschitz functions satisfying $[\pi]_{x}=\left[\pi^{\prime}\right]_{x}$. Then

$$
\left[f_{U \#} \pi\right]_{f(x)}=\left[f_{U^{\prime} \#} \pi^{\prime}\right]_{f(x)} .
$$

Proof. We may assume $U^{\prime} \subset U$. Since $[\pi]_{x}=\left[\pi^{\prime}\right]_{x}$, there exists $\rho>0$, for which $U(x, \rho) \subset U^{\prime}$ and

$$
\left.\left.\pi\right|_{U(x, \rho)^{k+1}} \equiv \pi^{\prime}\right|_{U(x, \rho)^{k+1}} .
$$

Since $U(x, \rho)$ is a normal neighborhood of $x$ we have, by the summation formula of the local index (3.2) that, for every $q \in B_{\rho}(p)$,

$$
U \cap f^{-1}(q)=U^{\prime} \cap f^{-1}(q)=U(x, \rho) \cap f^{-1}(q) .
$$

Thus

$$
\left.f_{U \#} \pi\right|_{B_{\rho}(p)^{k+1}}=\left.f_{U^{\prime} \#} \pi^{\prime}\right|_{B_{\rho}(p)^{k+1}},
$$

and

$$
\left[f_{U \#} \pi\right]_{f(x)}=\left[f_{U^{\prime} \#} \pi^{\prime}\right]_{f(x)} .
$$

The claim follows.

Definition 4.11. Let $f: X \rightarrow Y$ be a BLD-map between locally geodesic oriented cohomology manifolds. The local averaging map $A_{f}:$ Poly $^{k}(X) \rightarrow$ Poly $^{k}(Y)$ is the map

$$
[\pi]_{x} \mapsto \frac{1}{i_{f}(x)^{k+1}}\left[f_{U_{x} \#} \pi\right]_{f(x)}
$$

where, for each $x \in X, U_{x}$ is a normal neighborhood of $x$. 
By Lemma 4.10, the local averaging map $A_{f}: \mathcal{P o l y}^{k}(X) \rightarrow \mathcal{P o l y}^{k}(Y)$ is well-defined. Moreover, for each $x \in X$,

$$
A_{f}: \operatorname{Pol}_{x}^{k}(X) \rightarrow \operatorname{Pol}_{f}^{k}(x)(Y) \text {. }
$$

Remark 4.12. For each $x \in X$, the stalks $\mathcal{P o l}_{x}^{k}(X)$ and $\mathcal{P o l}_{f(x)}^{k}(Y)$ are vector spaces. We have, by the linearity of $f_{U \#}$ that, for $[\pi]_{x},\left[\pi^{\prime}\right]_{x} \in \operatorname{Pol}_{x}^{k}(X)$,

$$
A_{f}\left([\pi]_{x}+\left[\pi^{\prime}\right]_{x}\right)=A_{f}\left([\pi]_{x}\right)+A_{f}\left(\left[\pi^{\prime}\right]_{x}\right) .
$$

Definition 4.13. Let $\omega \in \mathscr{G}_{c}^{k}(X)$. The push-forward $f_{\#} \omega \in \mathscr{G}_{c}^{k}(Y)$ is the section

$$
f_{\#} \omega: Y \rightarrow \mathcal{P o}_{y}{ }^{k}(Y), \quad y \mapsto \sum_{x \in f^{-1}(y)} i_{f}(x) A_{f}(\omega(x)) .
$$

Note that, since $\omega \in \mathscr{G}_{c}^{k}(X)$ has compact support, the sum in Definition 4.13 has only finitely many nonzero summands.

Let $\omega \in \mathscr{G}_{c}^{k}(X)$ and $y \in Y$. The value of the push-forward $f_{\#} \omega$ at $y$ can be given as follows. Let $r>0$ be a radius with the property that $B_{r}(y)$ is a geodesic spread neighborhood with respect to spt $\omega$; cf. Lemma 3.1. For each $x \in f^{-1}(y) \cap \operatorname{spt} \omega$, let $\pi_{x} \in \operatorname{Poly}^{k}(U(x, r))$ satisfy $\left[\pi_{x}\right]_{x}=\omega(x)$. Then

$$
f_{\#} \omega(y)=\left[\left(A_{f}^{r} \omega\right)_{y}\right]_{y} \text {, where }\left(A_{f}^{r} \omega\right)_{y}=\sum_{x \in f^{-1}(y) \cap \operatorname{spt} \omega} \frac{1}{i_{f}(x)^{k}} f_{U(x, r) \#} \pi_{x} \in \operatorname{Poly}^{k}\left(B_{r}(y)\right) .
$$

Indeed, it suffices to note that

$$
\begin{aligned}
{\left[\left(A_{f}^{r} \omega\right)_{y}\right]_{y} } & =\sum_{x \in f^{-1}(y) \cap \operatorname{spt} \omega} \frac{1}{i_{f}(x)^{k}}\left[f_{U(x, r) \#} \pi_{x}\right]_{y} \\
& =\sum_{x \in f^{-1}(y) \cap \operatorname{spt} \omega} i_{f}(x) A_{f}\left(\left[\pi_{x}\right]_{x}\right)=\sum_{x \in f^{-1}(y)} i_{f}(x) A_{f}(\omega(x))=f_{\#} \omega(y) .
\end{aligned}
$$

We use this fact in the sequel.

The next proposition lists the basic properties of the push-forward.

Proposition 4.14. Let $f: X \rightarrow Y$ be an $L-B L D$ map. The pushforward $\omega \mapsto f_{\#} \omega$ is a linear map $f_{\#}: \mathscr{G}_{c}^{k}(X) \rightarrow$ $\mathscr{G}_{c}^{k}(Y)$ satisfying, for each $\omega \in \mathscr{G}_{c}^{k}(X)$, the following properties:

(1) $\operatorname{spt}\left(f_{\#} \omega\right) \subset f(\operatorname{spt} \omega)$,

(2) $L_{k}\left(f_{\#} \omega\right) \leq L^{k+1} f_{\#} L_{k}(\omega)$ and $\left\|f_{\#} \omega\right\| \leq L^{k} f_{\#}\|\omega\|$ pointwise on $Y$,

(4) $f_{\#}(d \omega)=d f_{\#} \omega$, and

(3) $f_{\#}\left(\alpha \smile f^{\#} \beta\right)=f_{\#} \alpha \smile \beta$ for $\alpha \in \mathscr{G}_{C}^{k}(X)$ and $\beta \in \mathscr{G}^{m}(Y)$.

Proof. Linearity is straighforward to check (see Remark 4.12). Let $\omega \in \mathscr{G}_{c}^{k}(X)$ and $p \in Y, p \notin f(\operatorname{spt} \omega)$. Then spt $\omega \cap f^{-1}(p)=\varnothing$ and therefore all the terms in the sum defining $f_{\#} \omega(p)$ are zero. This proves (1).

Let $B_{r}(p)$ be a geodesic spread neighborhood with respect to spt $\omega$. By Corollary 4.4 we have

$$
\begin{aligned}
L_{k}\left(A_{f}^{r} \omega_{p} ; B_{r}(p)\right) & \leq \sum_{x \in f^{-1}(p)} \frac{1}{i_{f}(x)^{k}} L^{k+1} i_{f}(x)^{k+1} L_{k}\left(\pi_{x} ; U(x, r)\right) \\
& =L^{k+1} \sum_{x \in f^{-1}(p)} i_{f}(x) L_{k}\left(\pi_{x} ; U(x, r)\right)
\end{aligned}
$$

where $\pi_{x} \in \operatorname{Poly}^{k}(U(x, r))$ satisfies $\left[\pi_{x}\right]_{x}=\omega(x)$ for $x \in \operatorname{spt} \omega \cap f^{-1}(p)$. Similarly

$$
\begin{aligned}
\operatorname{Lip}_{k}\left(f_{\#}^{r} \omega_{p} ; B_{r}(p)\right) & \leq \sum_{x \in f^{-1}(p)} \frac{1}{i_{f}(x)^{k}} L^{k} i_{f}(x)^{k+1} \operatorname{Lip}_{k}\left(\pi_{x} ; U(x, r)\right) \\
& =L^{k} \sum_{x \in f^{-1}(p)} i_{f}(x) \operatorname{Lip}_{k}\left(\pi_{x} ; U(x, r)\right) .
\end{aligned}
$$


Taking the limit $r \rightarrow 0$ yields (2).

To prove (3), we use Lemma 4.7. We have

$$
\begin{aligned}
A_{f}^{r}(d \omega)_{p} & =\sum_{x \in f^{-1}(p)} \frac{1}{i_{f}(x)^{k+1}} f_{U(x, r) \#}\left(d \pi_{x}\right)=\sum_{x \in f^{-1}(p)} \frac{i_{f}(x)}{i_{f}(x)^{k+1}} d f_{U(x, r) \#} \omega_{x} \\
& =d\left(\sum_{x \in f^{-1}(p)} \frac{1}{i_{f}(x)^{k}} f_{U(x, r) \#} \pi_{x}\right)=d A_{f}^{r} \omega_{p},
\end{aligned}
$$

for each $p \in Y$.

For (4), let $p \in Y$ and let $\beta_{p} \in \operatorname{Poly}^{k}\left(B_{r}(p)\right)$ be such that $\left[\beta_{p}\right]_{p}=\beta(p)$. For each $x \in \operatorname{spt} \omega \cap f^{-1}(p)$, choose polylipschitz functions $\alpha_{x} \in$ Poly $^{k}(U(x, r))$. By Lemma 4.6 we obtain

$$
\begin{aligned}
A_{f}^{r}\left(\alpha \smile f^{\#} \beta\right)_{p} & =\sum_{x \in f^{-1}(p)} \frac{1}{i_{f}(x)^{k+m}} f_{U(x, r) \#}\left(\alpha_{x} \smile f^{\#} \beta_{p}\right) \\
& =\sum_{x \in f^{-1}(p)} \frac{i_{f}(x)^{m}}{i_{f}(x)^{k+m}}\left(f_{U(x, r) \#} \alpha_{x}\right) \smile \beta_{p} \\
& =\left(\sum_{x \in f^{-1}(p)} \frac{1}{i_{f}(x)^{k}} f_{U(x, r) \#} \alpha_{x}\right) \smile \beta_{p}=\left(A_{f}^{r} \alpha_{p}\right) \smile \beta_{p}
\end{aligned}
$$

for each $p \in Y$. Thus

$$
f_{\#}\left(\alpha \smile f^{\#} \beta\right)(p)=\left[A_{f}^{r}\left(\alpha \smile f^{\#} \beta\right)_{p}\right]_{p}=\left[\left(A_{f}^{r} \alpha\right)_{p} \smile \beta_{p}\right]_{p}=\left(\left(f_{\#} \alpha\right) \smile \beta\right)(p),
$$

for each $p \in Y$.

\subsection{Partition-continuity of the push-forward of polylipschitz forms}

In general, $f_{\#} \omega$ need not be continuous for continuous $\omega \in \Gamma_{c}^{k}(X)$. However, $f_{\#}$ maps continuous sections to partition-continuous sections.

Proposition 4.15. Let $f: X \rightarrow Y$ be a BLD-map between locally geodesic, oriented cohomology manifolds. Suppose $\left(\pi_{0}, \ldots, \pi_{k}\right) \in \mathscr{D}^{k}(X), \pi=\pi_{0} \otimes \cdots \otimes \pi_{k} \in \operatorname{Poly}_{c}^{k}(X)$ and $\omega=\imath(\pi) \in \Gamma_{c}^{k}(X)$. Then there is a finite Borel partition $\left\{E_{i}\right\}_{j=1}^{N}$ of $Y$ for which

$$
\left.\left(f_{\#} \omega\right)\right|_{E_{i}} \in \Gamma^{k}\left(E_{i}\right)
$$

for each $j=1, \ldots, N$.

We prove Proposition 4.15 at the end of Section 4.2. For the proof, we briefly recall the monodromy representation of a proper branched covers.

Let $f: X \rightarrow Y$ be a proper branched cover. Then there is a locally compact geodesic space $X_{f}$, a finite group $G=G_{f}$, called the monodromy group of $f$, acting on $X_{f}$ by homeomorphisms, and a subgroup $H \leq G$ satisfying

$$
X_{f} / G \approx Y, U_{f} / H \approx X \text {. }
$$

The quotient maps

$$
\bar{f}: X_{f} \rightarrow Y, \quad x \mapsto G x,
$$

and

$$
\varphi: X_{f} \rightarrow X, \quad x \mapsto H x
$$


are branched covers for which the diagram

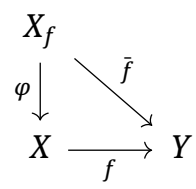

commutes. When $f$ is a BLD-map, the group $G$ acts on $X_{f}$ by bilipschitz maps, and $\bar{f}$ and $\varphi$ are BLD-maps. See [1] and the references therein for details on monodromy representations.

The following multiplicity formula is a counterpart of (3.4). We refer to [1] for similar multiplicity formulas.

Lemma 4.16. Let $f: X \rightarrow Y$ be a proper branched cover. Consider the monodromy triangle (4.3) associated to f. For $w \in X_{f}$, denote by $H_{w} \leq G_{w}$ the stabilizers of $w$ of $H$ and $G$, respectively. Then we have the identity

$$
\left|G_{w}\right|=i_{f}(\varphi(w))\left|H_{w}\right|
$$

for all $w \in X_{f}$.

Proof. Let $w \in X_{f}$ and let $W \subset X_{f}$ be a normal domain for $\bar{f}$. Then $\varphi(W) \subset X$ is a normal neighborhood of $\varphi(w)$ with respect to $f$. We denote $g=\left.(\bar{f})\right|_{W}: W \rightarrow \bar{f}(W)$. The stabilizers $G_{W}$ and $H_{w}$ act on $W$ and the restrictions $g$ and $\left.\varphi\right|_{W}$ are orbit maps with respect to the action. Thus, the commuting diagram

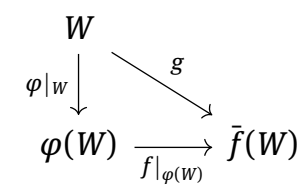

is a monodromy representation of $\left.f\right|_{\varphi(W)}$, with monodromy group $G_{W}$, and $\left.\varphi\right|_{W}$ is the orbit map for $H_{W}$. Since $g B_{g}$ and $f B_{f}$ are nowhere dense, there exists $p^{\prime} \in \bar{f} \backslash\left(g B_{g} \cup f B_{f}\right)$. Since $g$ is the orbit map for $G_{w}$, we have that

$$
\left|G_{w}\right|=\left|g^{-1}\left(p^{\prime}\right)\right| \text {. }
$$

On the other hand, $\left(\left.f\right|_{\varphi(W)}\right)^{-1}\left(p^{\prime}\right) \cap\left(\left.\varphi\right|_{W}\right) B_{\left.\varphi\right|_{W}}=\varnothing$. We conclude that

$$
\left|G_{W}\right|=\left|g^{-1}\left(p^{\prime}\right)\right|=\left|\left(\left.\varphi\right|_{W}\right)^{-1}\left(\left(\left.f\right|_{\varphi(W)}\right)^{-1}\left(p^{\prime}\right)\right)\right|=\left|H_{W}\right| \operatorname{deg}\left(\left.f\right|_{\varphi(W)}\right) .
$$

Since $\operatorname{deg}\left(\left.f\right|_{\varphi(W)}\right)=i_{f}(w)$, the claim follows.

\section{Fiber equivalence}

Throughout this subsection we fix a proper BLD-map $f: X \rightarrow Y$. We introduce the fiber equivalence on $Y$ using the monodromy representation (4.3) of $f$. Two points $p, q \in Y$ are said to be fiber equivalent, $p \sim_{f} q$, if $\left|f^{-1}(p)\right|=\left|f^{-1}(q)\right|$ and there are labelings of the preimages

$$
f^{-1}(p)=\left\{x_{1}, \ldots, x_{m}\right\} \quad \text { and } f^{-1}(q)=\left\{y_{1}, \ldots, y_{m}\right\}
$$

satisfying

$$
\left|\varphi^{-1}\left(x_{j}\right)\right|=\left|\varphi^{-1}\left(y_{j}\right)\right| \text { for each } j=1, \ldots, m .
$$

Lemma 4.17. The equivalence relation $\sim_{f}$ has finitely many equivalence classes, each of which is a Borel set.

Proof. The equivalence classes of $\sim_{f}$ are

$$
\left\{E_{m}\left(k_{1}, \ldots, k_{m}\right): m \in \mathbb{N}, k_{1}, \ldots, k_{m} \in \mathbb{N}\right\},
$$


where

$$
E_{m}\left(k_{1}, \ldots, k_{m}\right)=\left\{p \in Y:\left.f\right|_{U} ^{-1}(p)=\left\{x_{1}, \ldots, x_{m}\right\},\left|\varphi^{-1}\left(x_{j}\right)\right|=k_{j}, j=1, \ldots, m\right\}
$$

for each $m \in \mathbb{N}$ and $k_{1}, \ldots, k_{m} \in \mathbb{N}$. Since the sets $E_{m}\left(k_{1}, \ldots, k_{m}\right)$ are empty if $k_{j}>|G|$ or $m>\operatorname{deg} f$, we find that there are only finitely many equivalence classes of $\sim_{f}$.

To see that each of the sets $E_{m}\left(k_{1}, \ldots, k_{m}\right)$ is Borel, set

$$
E_{m}=\left\{p \in Y:\left|f^{-1}(p)\right|=m\right\}
$$

and

$$
E(k)=f(A(k)), A(k)=\left\{x \in X:\left|\varphi^{-1}(x)\right|=k\right\} .
$$

The sets $E_{m}$ and $E(k)$ are clearly Borel. Observe that

$$
E_{m}\left(k_{1}, \ldots, k_{m}\right)=E_{m} \cap E\left(k_{1}\right) \cap \cdots \cap E\left(k_{m}\right),
$$

whence the Borel measurability of the equivalence classes follows.

Lemma 4.18. If $p \sim_{f} q$, then $\left|\bar{f}^{-1}(p)\right|=\left|\bar{f}^{-1}(q)\right|$ and $i_{f}\left(x_{j}\right)=i_{f}\left(y_{j}\right)$ for each $j=1, \ldots, m$.

Proof. Let $z \in \bar{f}^{-1}(p)$ and $z^{\prime} \in \bar{f}^{-1}(q)$. Then

$$
\left|\bar{f}^{-1}(p)\right|=|G z|=\sum_{j}^{m}\left|\varphi^{-1}\left(x_{j}\right)\right|=\sum_{j}^{m}\left|\varphi^{-1}\left(y_{j}\right)\right|=\left|G z^{\prime}\right|=\left|\bar{f}^{-1}(q)\right| .
$$

For second claim let $H_{w} \leq G_{w}$ be the stabilizer subgroups of $w \in X_{f}$ in $H$ and $G$, respectively. Since $\varphi$ is an orbit map of the action $H \curvearrowright X_{f}$, we have that $|H w|=\left|\varphi^{-1} \varphi(w)\right|$. Hence

$$
\left|H_{w}\right|=\frac{|H|}{|H w|}=\frac{|H|}{\left|\varphi^{-1}(\varphi(w))\right|} .
$$

Thus, by Lemma 4.16, we have, for $w=z_{j} \in \varphi^{-1}\left(x_{j}\right)$ and $w=z_{j}^{\prime}=\varphi^{-1}\left(y_{j}\right)$, that

$$
i_{f}\left(x_{j}\right)=\frac{\left|G_{z_{j}}\right|}{\left|H_{z_{j}}\right|}=\frac{|G|}{\left|G_{z_{j}}\right|} \frac{\left|\varphi^{-1}\left(x_{j}\right)\right|}{|H|}=\frac{|G|}{|H|} \frac{\left|\varphi^{-1}\left(x_{j}\right)\right|}{\left|\bar{f}^{-1}(p)\right|}=\frac{|G|}{|H|} \frac{\left|\varphi^{-1}\left(y_{j}\right)\right|}{\left|\bar{f}^{-1}(q)\right|}=i_{f}\left(y_{j}\right)
$$

for each $j=1, \ldots, m$.

Lemma 4.19. Let $p \sim_{f} q$. Let $B_{r}(p)$ and $B_{s}(q)$ be spread neighborhoods for $f$ and $\bar{f}$. Then

$$
f^{-1}\left(B_{r}(p) \cap B_{s}(q)\right)=\bigcup_{j=1}^{m}\left(U_{f}\left(x_{j}, r\right) \cap U_{f}\left(y_{j}, s\right)\right) .
$$

Proof. For each $j=1, \ldots, m$ let $k_{j}=\left|\varphi^{-1}\left(x_{j}\right)\right|=\left|\varphi^{-1}\left(y_{j}\right)\right|$ and let

$$
\varphi^{-1}\left(x_{j}\right)=\left\{z_{j}^{1}, \ldots, z_{j}^{k_{j}}\right\}, \varphi^{-1}\left(y_{j}\right)=\left\{w_{j}^{1}, \ldots, w_{j}^{k_{j}}\right\} .
$$

Since $\left|G_{z_{j}}\right|=\left|G_{w_{j}^{l}}\right|$ for all $j=1, \ldots, m$ and $l=1, \ldots, k_{j}$, we have that

$$
\bar{f}^{-1}\left(B_{r}(p) \cap B_{s}(q)\right)=\bigcup_{j=1}^{m} \bigcup_{l=1}^{k_{j}}\left(U_{\bar{f}}\left(z_{j}^{l}, r\right) \cap U_{\bar{f}}\left(w_{j}^{l}, s\right)\right) .
$$

The claim now follows from the identity $f^{-1} A=\varphi\left(\bar{f}^{-1} A\right)$ for $A \subset Y$. 
Proof of Proposition 4.15. Suppose first that spt $\pi_{0} \subset U$ where $U \subset X$ is a normal domain for $f$. Then $f_{\#}(\omega)(p)=$ 0 whenever $p \in Y \backslash f(U)$.

Define the partition $\mathcal{E}$ on $f(U)$ as the collection of equivalence classes of the fiber equivalence relation $\sim:=\sim_{\left.f\right|_{U}}$ related to $\left.f\right|_{U}$.

Let $p, q \in f(U)$ be fiber equivalent, that is $p \sim q$, and let $B_{r}(p)$ and $B_{r}(q)$ be geodesic spread neighborhoods for $\left.f\right|_{U}$ and $\overline{\left.f\right|_{U}}$. Then, by Lemma 4.19,

$$
U_{f}\left(x_{j}, r\right) \cap\left(\left.f\right|_{U}\right)^{-1}(z)=U_{f}\left(y_{j}, s\right) \cap\left(\left.f\right|_{U}\right)^{-1}(z)
$$

for $j=1, \ldots, m$ and $z \in B_{r}(p) \cap B_{s}(q)$.

It follows that, for each $\bar{z}=\left(z_{0}, \ldots, z_{k}\right) \in\left(B_{r}(p) \cap B_{s}(q)\right)^{k+1}$, we have

$$
\left(\left.f\right|_{U\left(x_{j}, r\right)}\right)_{\#} \pi_{l}\left(z_{l}\right)=\left(\left.f\right|_{U\left(y_{j}, s\right)}\right)_{\#} \pi_{l}\left(z_{l}\right)
$$

for all $l=0, \ldots, k$. Thus, by Lemma 4.18,

$$
\begin{aligned}
A_{f}^{r} \omega_{p}(\bar{z}) & =\sum_{j=1}^{m} \frac{1}{i_{f}\left(x_{j}\right)^{k}}\left(\left.f\right|_{U\left(x_{j}, r\right)}\right)_{\#} \pi_{0}\left(z_{0}\right)\left(\left.f\right|_{U\left(x_{j}, r\right)}\right)_{\#} \pi_{1}\left(z_{1}\right) \cdots\left(\left.f\right|_{U\left(x_{j}, r\right)}\right)_{\#} \pi_{k}\left(z_{k}\right) \\
& =\sum_{j=1}^{m} \frac{1}{i_{f}\left(y_{j}\right)^{k}}\left(\left.f\right|_{U\left(y_{j}, s\right)}\right)_{\#} \pi_{0}\left(z_{0}\right)\left(\left.f\right|_{U\left(y_{j}, s\right)}\right)_{\#} \pi_{1}\left(z_{1}\right) \cdots\left(\left.f\right|_{U\left(y_{j}, s\right)}\right)_{\#} \pi_{k}\left(z_{k}\right) \\
& =A_{f}^{s} \omega_{q}(\bar{z})
\end{aligned}
$$

for all $\bar{z} \in\left(B_{r}(p) \cap B_{s}(q)\right)^{k+1}$.

For every $p \in U$ choose a radius $r_{p}>0$ such that $B_{r_{p}}(p)$ is a geodesic spread neighborhood for $f$ with respect to spt $\omega$. We have proved that $\left\{A_{f}^{r_{p}} \omega_{p}\right\}_{B_{r_{p}}(p)}$ satisfies the overlap condition (1) in [24, Definition 6.2] for every equivalence class of the fiber equivalence. Condition (2) in [24, Definition 6.2] follows from the first estimate in Corollary 4.4. Indeed, for each $p \in Y$, we have

$$
\begin{aligned}
& L_{k}\left(A_{f}^{r} \omega_{p} ; E \cap B_{r}(p)\right) \leq \sum_{x \in f^{-1}(p)} \frac{L^{k+1} i_{f}(x)^{k+1}}{i_{f}(x)^{k}} L_{k}\left(\pi_{0} \otimes \cdots \otimes \pi_{k} ; E \cap U(x, r)\right) \\
& \leq L^{k+1}\left(\sum_{x \in \operatorname{spt} \omega \cap f^{-1}(p)} i_{f}(x)\right) L\left(\left.\pi_{0}\right|_{B\left(\operatorname{spt} \pi_{0}, r\right)}\right) \cdots L\left(\left.\pi_{k}\right|_{B\left(\operatorname{spt} \pi_{0}, r\right)}\right) .
\end{aligned}
$$

By Lemma 4.17, $\mathcal{E}$ is a finite Borel partition. Thus $f_{\#} \omega \in \Gamma_{\mathrm{pc}, \mathrm{c}}^{k}(X)$.

We have demonstrated that $f_{\#} \omega$ is $\mathcal{E}$-continuous under the assumption that spt $\pi_{0}$ is contained in a normal domain for $f$. Suppose $\left(\pi_{0}, \ldots, \pi_{k}\right) \in \mathscr{D}^{k}(X)$ and let $\omega=\imath\left(\pi_{0} \otimes \cdots \otimes \pi_{k}\right) \in \Gamma_{c}^{k}(X)$. Set $K=\operatorname{spt} \omega$. Let $\mathcal{U}=\left\{U_{1}, \ldots, U_{M}\right\}$ be a finite covering of $K$ by normal domain for $f$ and let $\left\{\varphi_{1}, \ldots, \varphi_{M+1}\right\}$ be a Lipschitz partition of unity subordinate to $U \cup\{X \backslash K\}$ satisfying spt $\varphi_{M+1} \subset X \backslash K$.

For each $l=1, \ldots, M+1, \operatorname{spt}\left(\varphi_{l} \pi_{0}\right)$ is contained in a normal domain for $f$. Thus $f_{\#}\left(\varphi_{l} \omega\right)$ is $\mathcal{E}$-continuous. We conclude that the finite sum

$$
f_{\#} \omega=\sum_{l=1}^{M} f_{\#}\left(\varphi_{l} \omega\right)
$$

is $\mathcal{E}$-continuous.

\subsection{Pull-back of currents of locally finite mass by BLD-maps}

To define the pull-back of a $k$-current $T \in M_{k, l o c}(X)$ as $T \circ f_{\#}$ (see the discussion in the introduction) it remains to show that the resulting functional is weakly continuous.

Proposition 4.20. Let $f: X \rightarrow Y$ be a BLD-map between locally geodesic, oriented cohomology manifolds. If $\pi^{n} \rightarrow \pi$ in Poly $_{c}^{k}(X)$ then $f_{\#} \pi^{n} \rightarrow f_{\#} \pi$ in $\Gamma_{\mathrm{pc}, \mathrm{c}}^{k}(X)$. 
Proof. Let $K \subset X$ be a compact set containing spt $\pi_{0}^{n}$ for each $n \in \mathbb{N}$. Let $\mathcal{U}=\left\{U_{1}, \ldots, U_{M}\right\}$ be an open cover of $K$ by normal neighborhoods, and let $\left\{\varphi_{1}, \ldots, \varphi_{M+1}\right\}$ be a Lipschitz partition of unity subordinate to $\{X \backslash K\} \cup \mathcal{U}$. It suffices to prove

$$
f_{\#}\left(\varphi_{l} \smile \pi^{n}\right) \rightarrow f_{\#}\left(\varphi_{l} \smile \pi\right)
$$

in $\Gamma_{\mathrm{pc}, \mathrm{c}}^{\mathrm{k}}(X)$ for each $l=0, \ldots, M$.

Fix $U=U_{l}$ and let $\mathcal{E}=\left\{E_{1}, \ldots, E_{N}\right\}$ be the equivalence classes of the fiber equivalence $\sim_{\left.f\right|_{U}}$ associated to $\left.f\right|_{U}$. Let $\left\{B_{1}, \ldots, B_{Q}\right\}$ be a finite cover of $\overline{f U}$ such that each $B_{i}=B_{r_{i}}\left(p_{i}\right)$ is a geodesic spread neighborhood of $\left.f\right|_{U}$. For any $E=E_{m}\left(\mu_{1}, \ldots, \mu_{m}\right) \in \mathcal{E}$, we may write $\left.f\right|_{U} ^{-1}\left(p_{i}\right)=\left\{x_{1}, \ldots, x_{m}\right\}$, where $i_{f}\left(x_{l}\right)=\mu_{l}$; see the proof of Lemma 4.17. Set

$$
\sigma^{n}=\varphi_{U} \smile\left(\pi^{n}-\pi\right) .
$$

We have

$$
A_{f}^{r_{i}}\left(\sigma^{n}\right)=\sum_{l=1}^{m} \frac{1}{i_{f}\left(x_{l}\right)^{k}} f_{U\left(x_{l}, r_{i}\right) \#} \sigma^{n} .
$$

Since $\sigma^{n} \rightarrow 0$ in $\operatorname{Poly}_{U}^{k}(X)$, and hence the restrictions converge in Poly ${ }^{k}\left(U\left(x_{l}, r_{i}\right)\right)$, it follows from Lemma 4.8 that $\left.f_{\#}^{i}\left(\sigma^{n}\right)\right|_{\left(E \cap B_{i}\right)^{k+1}} \rightarrow 0$ in Poly ${ }^{k}\left(E \cap B_{i}\right)$. Furthermore,

$$
L_{k}\left(f_{\#}^{i} \sigma^{n} ; E \cap B_{i}\right) \leq \sum_{l=1}^{m} \frac{L^{k+1} \mu_{f}\left(U\left(x_{l}, r_{i}\right)\right)}{\mu_{l}^{k}} L_{k}\left(\sigma^{n} ; B_{i}\right) \leq L^{k+1} \mu_{f}(U) \sup _{n \in \mathbb{N}} L_{k}\left(\sigma^{n} ; U\right)
$$

for all $i \in \mathbb{N}$ and $E \in \mathcal{E}$. This shows that $f_{\#}\left(\varphi_{U} \smile \pi^{n}\right) \rightarrow f_{\#}\left(\varphi_{U} \smile \pi\right)$ in $\Gamma_{\mathrm{pc}, \mathrm{c}}^{k}(X)$. The claim follows.

We now define the pull-back of currents of locally finite mass.

Definition 4.21. Let $f: X \rightarrow Y$ be a BLD-map between two locally geodesic, oriented cohomology manifolds $X$ and $Y$, and let $T \in M_{k, \text { loc }}(Y)$ be a k-current of locally finite mass on $Y$. The pullback $f^{\star} T \in \mathscr{D}^{k}(X)$ of $T$ is the $k$-current

$$
\left(\pi_{0}, \ldots, \pi_{k}\right) \mapsto \widehat{T}\left(f_{\#}\left(\pi_{0} \otimes \cdots \otimes \pi_{k}\right)\right)
$$

for every $\left(\pi_{0}, \ldots, \pi_{k}\right) \in \mathscr{D}^{k}(X)$

Proposition 4.22. Let $f: X \rightarrow Y$ be an L-BLD-map between locally geodesic, oriented cohomology manifolds, and let $T \in M_{k, \text { loc }}(Y)$. Then $f^{\star} T \in M_{k, \text { loc }}(X)$ and

$$
\left\|f^{\star} T\right\| \leq L^{k} f^{\star}\|T\| .
$$

Proof. By Lemma 4.20 and Theorem 2.6, $f^{\star} T$ is sequentially continuous. Let $\left(\pi_{0}, \ldots, \pi_{k}\right) \in \mathscr{D}^{k}(X)$ and $\pi=$ $\pi_{0} \otimes \cdots \otimes \pi_{k}$. Suppose $E_{1}, \ldots, E_{N}$ is a Borel partition of $Y$ for which

$$
\left.f_{\#} \pi\right|_{E_{i}} \in \Gamma^{k}\left(E_{i}\right)
$$

for each $i=1, \ldots, N$. By [24, Proposition 6.7] and Lemma 4.14, we may estimate

$$
\begin{aligned}
\left|f^{\star} T(\pi)\right| & =\left|\widehat{T}\left(f_{\#} \pi\right)\right| \leq \sum_{i=1}^{N} \int_{E_{i}}\left\|\left.f_{\#} \pi\right|_{E_{i}}\right\| p \mathrm{~d}\|T\|(p) \leq \sum_{i=1}^{N} \int_{E_{i}} L^{k} f_{\#}\|\pi\|(p) \mathrm{d}\|T\|(p) \\
& =L^{k} \int_{Y} f_{\#}\|\pi\| \mathrm{d}\|T\|=L^{k} \int_{X}\|\pi\| \mathrm{d}\left(f^{\star}\|T\|\right) .
\end{aligned}
$$

This proves $f^{\star} T$ is a $k$-current of locally finite mass and provides the desired estimate. 
Proof of Theorem 1.1. Properties (1) and (2) follow directly from the corresponding properties (3) and (4) in Proposition 4.14. Indeed, let $T \in M_{k, \text { loc }}(Y)$. Then

$$
\begin{aligned}
f^{\star}(\partial T)(\pi) & =\widehat{\partial T}\left(f_{\#} \pi\right)=\partial \widehat{T}\left(f_{\#} \pi\right)=\widehat{T}\left(d f_{\#} \pi\right)=\widehat{T}\left(f_{\#}(d \pi)\right) \\
& =\partial\left(\widetilde{T} \circ f_{\#}\right)(\pi)=\partial\left(f^{\star} T\right)(\pi)
\end{aligned}
$$

and

$$
f_{\star}\left(\left.\left(f^{\star} T\right)\right|_{E}\right)(\sigma)=f^{\star} T\left(\chi_{E} \smile f^{\#} \sigma\right)=\widehat{T}\left(f_{\#}\left(\chi_{E} \smile f^{\#} \sigma\right)\right)=\widehat{T}\left(f_{\#} \chi_{E} \smile \sigma\right),
$$

for all $\pi \in \mathscr{D}^{k}(X), \sigma \in \mathscr{D}^{k}(Y)$ and Borel set $E \subset X$.

To establish (3), let $E \subset X$ be a Borel set. We use (2) together with [17, Lemma 4.6] to conclude that

$$
\|T\|\left\lfloor_{f_{\# X_{E}}}=\| T\left\lfloor_ { f _ { \sharp X _ { E } } } \| = \| f _ { \star } \left(\left(f^{\star} T\right)\left\lfloor_{E}\right) \| \leq L^{k} f_{\star}\left(\left\|f^{\star} T\right\|\left\lfloor_{E}\right) .\right.\right.\right.\right.
$$

Thus

$$
\int_{E} \mathrm{~d}\left(f^{\star}\|T\|\right)=\int_{Y} f_{\#} \chi_{E} \mathrm{~d}\|T\| \leq L^{k} \int_{X} \chi_{E} \mathrm{~d}\left\|f^{\star} T\right\|=L^{k} \int_{E} \mathrm{~d}\left\|f^{\star} T\right\| .
$$

Hence $f^{\star}\|T\|(E) \leq L^{k}\left\|f^{\star} T\right\|(E)$ for all Borel sets $E \subset X$. We conclude that

$$
f^{\star}\|T\| \leq L^{k}\left\|f^{\star} T\right\| .
$$

The converse inequality is proven in Proposition 4.22. Naturality is proven in the next subsection, Proposition 4.26.

\subsection{Uniqueness and naturality of the pull-back}

We now turn our attention to the uniqueness and naturality of the pull-back. This section also contains the proof of Theorem 1.2.

Proposition 4.23. Let $f: X \rightarrow Y$ be a BLD-map between locally geodesic, oriented cohomology manifolds. Let $T \in M_{k, l o c}(X)$ be a $k$-current for which

$$
\left.f_{\star}(T\rfloor_{E}\right)=0
$$

for every precompact Borel set $E \subset X$. Then $T=0$.

We begin with an auxiliary lemma on BLD-maps.

Lemma 4.24. Let $f: X \rightarrow Y$ be a L-BLD-map between locally geodesic, oriented cohomology manifolds, and let $U \subset X$ be a normal domain for $f$. For each $m \in \mathbb{N}$, let

$$
E_{m}=U \cap i_{f}^{-1}(m) .
$$

Then $\left.f\right|_{E_{m}}$ is locally L-bilipschitz.

Proof. Let $x \in E_{m}$ and $r>0$ be such that $U(x, 2 L r) \subset U$ is a normal domain for $x$. We claim that $\left.f\right|_{E_{m} \cap U(x, r)}$ is $L$-bilipschitz.

First we show that $\left.f\right|_{E_{m} \cap U(x, 2 L r)}$ is injective. Let $y \in E_{m} \cap U(x, 2 L r)$. Then $m=i_{f}(y)=i_{f}(x)$. Suppose $f^{-1}(f(y)) \cap U(x, 2 L r) \neq\{y\}$. Then, by the summation formula (3.2), we have

$$
i_{f}(x)=\mu_{f}(U(x, 2 r L), x)=\mu_{f}(y, U(x, 2 r L))=\sum_{x^{\prime} \in f^{-1}(f(y)) \cap U(x, 2 L r)} i_{f}\left(x^{\prime}\right)>i_{f}(y),
$$

since $i_{f}\left(x^{\prime}\right) \geq 1$ for each $x^{\prime} \in X$. This is a contradiction, whence

$$
f^{-1}(f(y)) \cap U(x, 2 L r)=\{y\}
$$


and $\left.f\right|_{E_{m} \cap U(x, 2 L r)}$ is injective.

The fact that $\left.f\right|_{E_{m} \cap U(x, r)}$ is $L$-Lipschitz is clear. Moreover the proof of injectivity shows that

$$
E_{m} \cap U(x, r)=f^{-1}\left(f\left(E_{m} \cap U(x, r)\right)\right) .
$$

Let $z^{\prime}, w^{\prime} \in f\left(E_{m} \cap U(x, r)\right)$, and let $z, w \in E_{m} \cap U(x, r)$ satisfy $z^{\prime}=f(z)$ and $w^{\prime}=f(w)$. Suppose $y$ is a geodesic joining $z^{\prime}$ and $w^{\prime}$ in $B_{2 r}(f(x))$. Since $f^{-1}\left(w^{\prime}\right) \cap U(x, r)=\{w\}$, we have that a lift $y^{\prime}$ in $U(x, 2 L r)$ of $y$ starting at $z$ ends at $w$. Thus

$$
d(z, w) \leq \ell\left(y^{\prime}\right) \leq L \ell(f \circ y)=L \ell(y)=L d\left(z^{\prime}, w^{\prime}\right) .
$$

This finishes the proof of the claim.

Proof of Proposition 4.23. Let $x \in X$. If $x \notin B_{f}$, let $r>0$ be such that $\left.f\right|_{B_{r}(x)}$ is bilipschitz. Then

$$
T\left\lfloor_{B_{r}(x)}=\left(\left.f\right|_{B_{r}(x)} ^{-1}\right) \star\left(\left.f\right|_{B_{r}(x)}\right) \star\left(T\left\lfloor_{B_{r}(x)}\right)=\left(\left.f\right|_{B_{r}(x)} ^{-1}\right) \star f_{\star}\left(T\left\lfloor_{B_{r}(x)}\right)=0 .\right.\right.\right.
$$

Suppose now that $x \in B_{f}$. Let $r>0$ be a radius for which $U(x, L r)$ is a normal neighborhood of $x$ and for which $\left.f\right|_{U(x, r) \cap i_{f}^{-1}(m)}$ is $L$-bilipschitz. Then

$$
B_{r}(x)=\bigcup_{m=1}^{i_{f}(x)}\left(E_{m} \cap B_{r}(x)\right),
$$

where $E_{m}=U(x, L r) \cap i_{f}^{-1}(m)$. Let $m \leq i_{f}(x)$. By the same argument as above

$$
T\left\lfloor_{E_{m} \cap B_{r}(x)}=\left(\left.f\right|_{E_{m} \cap B_{r}(x)}\right)^{-1} f_{\star}\left(T\left\lfloor_{E_{m} \cap B_{r}(x)}\right)=0 .\right.\right.
$$

Thus

$$
T \bigsqcup_{B_{r}(x)}=\sum_{m=1}^{i_{f}(x)} T\left\lfloor_{E_{m} \cap B_{r}(x)}=0 .\right.
$$

We have proven that, for each $x \in X$, there exists a radius $r>0$ such that

$$
T\left\lfloor_{B_{r}(x)}=0 .\right.
$$

Thus $T=0$.

Proof of Theorem 1.2. By Theorem 1.1 (1) and the assumption on $S$, we have

$$
f_{\star}\left[\left(f^{\star} T-S\right)\lfloor E]=0\right.
$$

for every precompact Borel set $E \subset X$. Proposition 4.23 implies that

$$
f^{\star} T-S=0,
$$

completing the proof.

We now prove the naturality of the pull-back. The first auxiliary result is the naturality of the push-forward of polylipschitz forms.

Proposition 4.25. Let $f: X \rightarrow Y$ and $g: Y \rightarrow Z$ be BLD-maps between locally geodesic, oriented cohomology manifolds $X, Y$ and $Z$. Let $\left(\pi_{0}, \ldots, \pi_{k}\right) \in \mathscr{D}^{k}(X)$ and $\pi=\pi_{0} \otimes \cdots \otimes \pi_{k} \in \operatorname{Poly}_{c}^{k}(X)$. Then

$$
g_{\#}\left(f_{\#} \pi\right)=(g \circ f)_{\#} \pi
$$

as sections in $\mathscr{G}_{c}^{k}(Z)$. 
Proof. The composition $g \circ f: X \rightarrow Z$ is a BLD-map. Denote by $L$ be the maximum of the BLD constans of $f$ and $g$. Let $\left(\pi_{0}, \ldots, \pi_{k}\right) \in \mathscr{D}^{k}(X)$ and $\pi=\pi_{0} \otimes \cdots \otimes \pi_{k} \in \operatorname{Poly}_{c}^{k}(X)$. Denote $\omega=f_{\#} \pi \in \mathscr{G}_{c}^{k}(Y)$, and spt $\pi=K$. Let $q \in Z$ and fix $r>0$ for which $B_{r}(q)$ is a geodesic spread neighborhood for $g \circ f$ with respect to $K$, and $U_{g}(y, r)$ is a geodesic spread neighborhood of $y$, for each $y \in g^{-1}(q) \cap f(K)$, with respect to $K$. Since

$$
(g \circ f)^{-1}\left(B_{r}(q)\right)=\bigcup_{y \in g^{-1}(q)} f^{-1}\left(U_{g}(y, r)\right)=\bigcup_{x \in(g \circ f)^{-1}(q)} U_{g \circ f}(x, r),
$$

we have that, for each $y \in g^{-1}(q) \cap f(K)$, the set $f^{-1}\left(U_{g}(y, r)\right)$ is a pairwise disjoint union

$$
f^{-1}\left(U_{g}(y, r)\right)=\bigcup_{x \in f^{-1}(y)} U_{g \circ f}(x, r) .
$$

Thus, each $U_{g \circ f}(x, r)$ is a normal neighborhood of $x$ with respect to $f$. For each $y \in g^{-1}(q) \cap f(K)$, let

$$
\sigma_{y}=\left(A_{f}^{r} \pi\right)_{y}=\sum_{x \in f^{-1}(y) \cap K} \frac{1}{i_{f}(x)^{k}} f_{U_{g \circ f}(x, r) \# ;}
$$

see the discussion after Definition 4.13. Then $g_{\#}\left(f_{\#} \pi\right)(q)=\left[A_{g}^{r} \omega_{q}\right]_{q}$, where

$$
A_{g}^{r} \omega_{q}=\sum_{y \in g^{-1}(q) \cap f(K)} \frac{1}{i_{g}(y)^{k}} g_{U_{g}(y, r) \#} \sigma_{y} .
$$

For each $y \in f^{-1}(q) \cap f(K)$ we have, by Lemma 4.9, that

$$
\begin{aligned}
g_{U_{g}(y, r) \#} \omega_{y} & =\sum_{x \in f^{-1}(y) \cap K} \frac{1}{i_{f}(x)^{k}} g_{U_{g}(y, r) \#} f_{U_{g \circ f}(x, r) \#} \pi \\
& =\sum_{x \in f^{-1}(y) \cap K} \frac{1}{i_{f}(x)^{k}}(g \circ f)_{U_{g \circ f}(x, r) \#} \pi .
\end{aligned}
$$

Thus we have

$$
\begin{aligned}
A_{g}^{r} \omega_{q} & =\sum_{y \in g^{-1}(q) \cap f(K)} \sum_{x \in f^{-1}(y) \cap K} \frac{1}{i_{g}(y)^{k} i_{f}(x)^{k}}(g \circ f)_{U_{g \circ f}(x, r) \#} \pi \\
& =\sum_{y \in g^{-1}(q) \cap f(K)} \sum_{x \in f^{-1}(y) \cap K} \frac{1}{i_{g \circ f}(x)^{k}}(g \circ f)_{U_{g \circ f}(x, r) \#} \pi \\
& =\sum_{x \in g \circ f^{-1}(q) \cap K} \frac{1}{i_{g \circ f}(x)^{k}}(g \circ f)_{U_{g \circ f}(x, r) \#} \pi=A_{g \circ f}^{r} \pi
\end{aligned}
$$

on $B_{r}(q)^{k+1}$. From this we conclude that

$$
g_{\#}\left(f_{\#} \pi\right)(q)=(g \circ f)_{\#} \pi(q)
$$

for all $q \in Z$.

Proposition 4.25 yields the naturality of the push-forward $f_{\#}: \mathscr{G}_{c}^{k}(X) \rightarrow \mathscr{G}_{c}^{k}(Y)$ for a BLD map $f: X \rightarrow Y$. We use this and Theorem 1.2 to conclude the naturality of the pull-back of metric currents.

Proposition 4.26. Let $f: X \rightarrow Y$ and $g: Y \rightarrow Z$ be BLD-maps between locally geodesic, oriented cohomology manifolds. Then

$$
(g \circ f)^{\star}=f^{\star} \circ g^{*}
$$

as maps $M_{k, l o c}(Z) \rightarrow M_{k, l o c}(X)$.

Note that, if $T \in M_{k, l o c}(Z)$, then, by definition and Proposition 4.25, we have

$$
(g \circ f)^{\star} T(\pi)=T\left((g \circ f)_{\#} \pi\right)=T\left(g_{\#} f_{\#} \pi\right)
$$

for $\pi \in \mathscr{D}^{k}(X)$. Unfortunately, since $f_{\#} \pi \in \mathscr{G}_{c}^{k}(Y)$ is not necessarily in $\Gamma_{c}^{k}(Y)$, we cannot conclude that $g^{\star} T\left(f_{\#} \pi\right)$ (strictly speaking, $\widehat{g^{\star} T}\left(f_{\#} \pi\right)$ ) is given by $T\left(g_{\#} f_{\#} \pi\right)$. 
Proof. We use Theorem 1.2. Let $\eta \in \operatorname{LIP}_{c}(X)$ and $\pi \in \mathscr{D}^{k}(Z)$. Then, by [24, Lemma 4.14 (a)], Proposition 4.14 (4) and Proposition 4.25,

$$
\begin{aligned}
(g \circ f)_{\star}\left(f^{\star} g^{\star} T\lfloor\eta)(\pi)\right. & =\left(f^{\star} g^{\star} T\right)\left(\eta \smile(g \circ f)^{\#} \pi\right)=T\left(g_{\#} f_{\#}\left(\eta \smile f^{\#} g^{\#} \pi\right)\right) \\
& =T\left(g_{\#}\left(f_{\#} \eta \smile g^{\#} \pi\right)\right)=T\left(g_{\#} f_{\#} \eta \smile \pi\right) \\
& =\left(T\left\lfloor(g \circ f)_{\#} \eta\right)(\pi) .\right.
\end{aligned}
$$

Let $E \subset X$ be a Borel set. Let $\eta_{j}$ be a sequence in $\operatorname{LIP}_{c}(X)$ converging to $\chi_{E}$ in $L^{1}(\|T\|)$ and $\|T\|$-almost everywhere; see [12, Proposition 2.3.13 and Remark 2.3.16(a)]. Then, for each $\pi \in \mathscr{D}^{k}(Z)$,

$$
\begin{aligned}
& (g \circ f)_{\star}\left(f^{\star} g^{\star} T\lfloor E)(\pi)=\lim _{n \rightarrow \infty}(g \circ f) \star\left(f^{\star} g^{\star} T\left\lfloor\eta_{n}\right)(\pi)\right.\right. \\
& =\lim _{n \rightarrow \infty}\left(T\left\lfloor(g \circ f)_{\#} \eta_{n}\right)(\pi)=T\left\lfloor(g \circ f)_{\# X_{E}}(\pi) .\right.\right.
\end{aligned}
$$

Thus, by Theorem 1.2, we have that

$$
f^{\star} g^{\star} T=(g \circ f)^{\star} T .
$$

This completes the proof.

\subsection{Pull-back of proper BLD maps}

Throughout this subsection, $f: X \rightarrow Y$ is a proper $L$-BLD map between geodesic, oriented cohomology manifolds $X$ and $Y$. Recall that a proper branched cover is $(\operatorname{deg} f)$-to-one; see the discussion on the branch set in Section 6.3.

In this subsection we prove Corollary 1.4, that is, we prove that the pull-back $f^{\star}: M_{k, \text { loc }}(Y) \rightarrow M_{k, \text { loc }}(X)$ satisfies the following properties:

1. the composition $f_{\star} \circ f^{\star}: M_{k, \text { loc }}(Y) \rightarrow M_{k, \text { loc }}(Y)$ satisfies $f_{\star} \circ f^{\star}=(\operatorname{deg} f)$ id;

2. the pull-back $f^{\star}$ commutes with the boundary, i.e. $\partial f^{\star} T=f^{\star}(\partial T)$ for $T \in M_{k, \text { loc }}(X)$; and

3. for each $T \in M_{k}(X)$,

$$
\frac{1}{L^{k}}(\operatorname{deg} f) M(T) \leq M\left(f^{\star} T\right) \leq L^{k}(\operatorname{deg} f) M(T) .
$$

Moreover, the pull-back operator $f^{\star}$ restricts to an operator

$$
f^{\star}: N_{k}(Y) \rightarrow N_{k}(X) .
$$

Proof of Corollary 1.4. Besides the restriction claim, we only need to prove (1) and (3) in the claim of Theorem 1.4. Let us first prove (3).

Let $T \in M_{k}(Y)$ be a current of finite mass. For any compact set $K \subset X$, Theorem 1.1 (3) implies the estimate

$$
L^{-k} \int_{Y} f_{\#} \chi_{K} \mathrm{~d}\|T\| \leq\left\|f^{\star} T\right\|(K) \leq L^{k} \int_{Y} f_{\#} \chi_{K} \mathrm{~d}\|T\| .
$$

Let $\left(K_{j}\right)$ be an increasing sequence of compact sets in $X$ for which

$$
\bigcup_{j \in \mathbb{N}} K_{j}=X .
$$

Then

$$
f_{\# X_{K_{j}}} \rightarrow f_{\# \chi_{X}}=\operatorname{deg} f
$$

pointwise. Thus

$$
\lim _{j \rightarrow \infty} \int_{Y} f_{\#} \chi_{K_{j}} \mathrm{~d}\|T\|=(\operatorname{deg} f) \int_{Y} \mathrm{~d}\|T\|=(\operatorname{deg} f) M(T) .
$$


This proves (3).

By (4.4) and Theorem 1.1(1), we have

$$
f_{\star}\left(f^{\star} T\left\lfloor\chi_{K_{j}}\right)=T \mid f_{\#} \chi_{K_{j}} \rightarrow(\operatorname{deg} f) T\right.
$$

weakly in $M_{k, \text { loc }}(X)$ as $j \rightarrow \infty$. On the other hand

$$
f^{\star} T\left\lfloor\chi_{K_{j}} \rightarrow f^{\star} T\right.
$$

weakly in $M_{k, \text { loc }}(X)$ as $j \rightarrow \infty$. Thus (1) in Corollary 1.4 is proven.

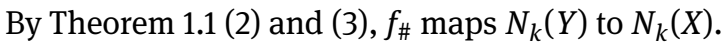

\section{Equidistribution estimates for pull-back currents}

\subsection{BLD-maps from $\mathbb{R}^{n}$ into metric spaces}

Let $X$ be a metric space and $f: \mathbb{R}^{n} \rightarrow X$ a Lipschitz map. We will use the metric Jacobian $J f$ of $f$, defined by Kirhchheim [16]: for almost every $x \in \mathbb{R}^{n}$ the limit

$$
\lim _{h \rightarrow 0} \frac{d(f(x+h v), f(x))}{|h|}, v \in \mathbb{R}^{n}
$$

exists for all $v \in \mathbb{R}^{n}$ and defines a seminorm. The metric differential

$$
\operatorname{md}_{x} f: \mathbb{R}^{n} \rightarrow[0, \infty)
$$

of $f$ at such a point $x$ is the seminorm given by (5.1) and zero otherwise. This induces the metric Jacobian If $: \mathbb{R}^{n} \rightarrow \mathbb{R}$, a Borel function defined for any point where the limit (5.1) exists, by

$$
J f(x)=\left(\int_{S^{n-1}} \operatorname{md}_{x} f(v)^{-n} \mathrm{~d} \sigma_{n-1}(v)\right)^{-1} .
$$

Here $\sigma_{n-1}$ is the normalized surface measure on the unit sphere $S^{n-1}$ of $\mathbb{R}^{n}$. The metric Jacobian plays a prominent role in the co-area formula

$$
\int_{\mathbb{R}^{n}} g J f \mathrm{~d} x=\int_{X}\left(\sum_{x \in f^{-1}(y)} g(x)\right) \mathrm{d} \mathcal{H}^{n}(y) .
$$

We refer to [16] for details.

Remark 5.1. By [18, Lemma 2.4] we obtain that, if the limit in (5.1) exists for $x \in \mathbb{R}^{n}$, then

$$
L^{-1} \leq \operatorname{md}_{x}(v) \leq L
$$

for any $v \in S^{n-1}$.

Remark 5.1 together with the co-area formula (5.2) implies that a BLD-elliptic oriented cohomology $n$-manifold is locally Ahlfors $n$-regular; see [13, Proposition 6.3 and Remark 4.16(b)].

Throughout the rest of this section $X$ is a compact geodesic oriented cohomology $n$-manifold, and $f: \mathbb{R}^{n} \rightarrow X$ an $L$-BLD map. We denote

$$
|X|=\mathcal{H}^{n}(X) \text { and } D=\operatorname{diam}(X) .
$$

By Remark 5.1 and the discussion after it the space $X$ is Ahlfors $n$-regular under the present assumptions. In particular $|X| \leq C D^{n}$, where $C>0$ is the Ahlfors regularity constant. 


\subsection{Equidistribution}

We turn our attention to the value distribution of BLD-maps. The following theorem will be used in the next subsection to obtain estimates on the mass of pullbacks of currents. For the theorem, let $A_{f}:(0, \infty) \rightarrow(0, \infty)$ be the function

$$
R \mapsto \frac{1}{|X|} \int_{B(R)} J f \mathrm{~d} x,
$$

and denote $B(R)=B(0, R) \subset \mathbb{R}^{n}$.

Theorem 5.2. Let $f: \mathbb{R}^{n} \rightarrow X$ be an L-BLD-mapping to a compact geodesic oriented cohomology manifold. Then there exists a constant $c(n, L)>0$ for which

$$
\left(1-\frac{c(n, L) D}{R}\right) \leq \frac{f_{\# \chi_{B(R)}}(p)}{A_{f}(R)} \leq\left(1+\frac{c(n, L) D}{R}\right)
$$

for every $p \in X$ and $R \geq L D$.

Theorem 5.2 gives a quantitative equidistribution estimate with constants depending only on $n$ and $L$. We refer to [20] and [23] for similar results for quasiregular maps. We begin with an observation which we record as a lemma.

Lemma 5.3. For a compactly supported Borel function $h: \mathbb{R}^{n} \rightarrow \mathbb{R}$, we have

$$
\int_{X} f_{\#} h \mathrm{~d} \mathcal{H}^{n}=\int_{\mathbb{R}^{n}} h J f \mathrm{~d} x .
$$

Proof. By [22, Corollary 10.2] we have $\left|f^{-1} f B_{f}\right|=0$. Thus

$$
f_{\#} h(y)=\sum_{x \in f^{-1}(y)} h(x)
$$

for almost every $y \in \mathbb{R}^{n}$. The rest follows directly from the change of variables formula (5.2).

Proof of Theorem 5.2. Let $R>L D$, and let $\chi_{B(R-\delta)} \leq \eta \leq \chi_{B(R)}$ be a Lipschitz function. By Lemma 3.3, for points $p, q \in X \backslash f B_{f}$, there is a bijection

$$
\psi: f^{-1}(q) \rightarrow f^{-1}(p)
$$

satisfying

$$
d(p, q) / L \leq|x-\psi(x)| \leq L d(p, q)
$$

for all $x \in f^{-1}(q) \cap B(R)$. Thus

$$
f_{\star} \eta(p)-f_{\star} \eta(q) \leq \sum_{x \in f^{-1}(q)}|\eta(\psi(x))-\eta(x)| .
$$

We have $|\eta(\psi(x))-\eta(x)| \leq 1$ for all $x$. Moreover,

(i) $|\eta(\psi(x))-\eta(x)|=0$, if $x \in B(R-L D-\delta)$, and

(ii) $|\eta(\psi(x))-\eta(x)|=0$, if $x \in \mathbb{R}^{n} \backslash B(R+L D)$.

Therefore

$$
\sum_{x \in f^{-1}(q)}|\eta(\psi(x))-\eta(x)| \leq \sum_{x \in f^{-1}(q)} \chi_{B(R+L D) \backslash B(R-L D-\delta)}(x)
$$

and we obtain

$$
f_{\#} \eta(p) \leq f_{\# \chi_{B(R)}}(q)+f_{\#} \chi_{B(R+L D) \backslash B(R-L D-\delta)}(q)
$$


for almost every $q$. Denote

$$
A(t, s)=B(t) \backslash B(s), \quad \text { for } t>s .
$$

Integrating with respect to $q$ we obtain

$$
\begin{aligned}
f_{\#} \eta(p) & \leq \int_{X} f_{\#}\left(\chi_{B(R)}+\chi_{B(R+L D) \backslash B(R-L D-\delta)}\right) \mathrm{d} q \\
& =\frac{1}{|X|}\left(\int_{B(R)} J f \mathrm{~d} x+\int_{A(R+L D, R-L D-\delta)} J f \mathrm{~d} x\right)
\end{aligned}
$$

In similar fashion we may obtain the estimate

$$
f_{\#} \eta(p)-f_{\#} \eta(q) \leq f_{\#} \chi_{A(R+L D, R-L D-\delta)}(p) .
$$

Fixing $q$ and integrating with respect to $p$ yields

$$
f_{X} f_{\star} \eta \mathrm{d} p \leq f_{\star} \eta(q)+\int_{X} f_{\#} \chi_{A(R+L D, R-L D-\delta)} \mathrm{d} p,
$$

or

$$
\frac{1}{|X|}\left(\int_{B(R-\delta)} J f \mathrm{~d} x-\int_{A(R+L D, R-L D-\delta)} J f \mathrm{~d} x\right) \leq f_{\#} \eta(q) .
$$

Since $f_{\#} \eta$ is continuous, estimates (5.4) and (5.5) hold for all $p \in Y$. Letting $\delta \rightarrow 0$ we obtain

$$
1-\frac{1}{|X| A_{f}(R)} \int_{A(R+L D, R-L D)} J f \leq \frac{f_{\# \chi_{B(R)}(p)}}{A_{f}(R)} \leq 1+\frac{1}{|X| A_{f}(R)} \int_{A(R+L D, R-L D)} J f
$$

for all $p \in M$.

Furthermore

$$
\frac{1}{|X| A_{f}(R)} \int_{A(R+L D, R-L D)} J f \leq \frac{L^{n} 2 L D n(R+L D)^{n-1}}{L^{-n} R^{n}} \leq \frac{2^{n} n L^{2 n+1} D}{R} .
$$

This implies the claim.

\subsection{Mass and flat norm estimates}

We apply the equidistribution Theorem 5.2 to prove estimates for the mass and flat norm of pull-backs of locally normal currents.

Theorem 5.4. Let $f: \mathbb{R}^{n} \rightarrow X$ be an L-BLD map into a compact, geodesic oriented cohomology $n$-manifold $X$. Let $T \in N_{k}(X)$ and $R>C D$, where $C$ is the constant in Theorem 5.2. Then there is a constant $c=c(n, L, k)$ for which

$$
\frac{1}{c} A_{f}(R) \mathcal{F}(T) \leq \mathcal{F}_{B_{R}}\left(f^{\star} T\right) \leq c A_{f}(R) \mathcal{F}(T)
$$

and

$$
\frac{1}{c} A_{f}(R) M(T) \leq\left\|f^{\star} T\right\|(B(R)) \leq c A_{f}(R) M(T) .
$$

Proof. Denote $\chi_{B(R)}=: \chi_{R}$. By Theorem 5.2 we have

$$
\frac{1}{2} \leq \frac{f_{\#} \chi_{R}}{A_{f}(R)} \leq 2
$$


for $R>2 C(n, L) D$. Thus

$$
\begin{aligned}
\frac{1}{2} L^{-k} A_{f}(R) M(T) & \leq L^{-k} \int_{X} f_{\# \chi_{R}} \mathrm{~d}\|T\|=L^{-k} f^{\star}\|T\|(B(R)) \\
& \leq\left\|f^{\star} T\right\|(B(R)) \leq L^{k} f^{\star}\|T\|(B(R)) \\
& =L^{k} \int_{X} f_{\#} \chi_{R} \mathrm{~d}\|T\| \leq 2 L^{k} A_{f}(R) M(T),
\end{aligned}
$$

establishing the first estimate.

To estimate the flat norm, let $A \in N_{k+1}(X)$. Then, by Proposition 4.22, we have that

$$
\begin{aligned}
\left\|f^{\star} T-\partial f^{\star} A\right\|\left(B_{R}\right) & +\left\|f^{\star} A\right\|\left(B_{R}\right) \\
& =\left\|f^{\star}(T-\partial A)\right\|\left(B_{R}\right)+\left\|f^{\star} A\right\|\left(B_{R}\right) \\
& \leq 2 L^{k} A_{f}(R) M(T-\partial A)+2 L^{k+1} A_{f}(R) M(A) \\
& \leq 2 L^{k+1} A_{f}(R)(M(T-\partial A)+M(A)) .
\end{aligned}
$$

Thus

$$
\mathcal{F}_{B(R)}\left(f^{\star} T\right) \leq 2 L^{k+1} A_{f}(R) \mathcal{F}(T) .
$$

For the opposite inequality, let $\eta: \mathbb{R}^{n} \rightarrow[0, \infty), \eta(x)=\left(1-\operatorname{dist}\left(B_{R-1}, x\right)\right)_{+}$be a Lipschitz function. By the proof of Proposition 1.1 (2) we have

$$
T\left\lfloor f_{\star} \eta=f_{\star}\left(\left(f^{\star} T\right)\lfloor\eta)\right.\right.
$$

Let $\varphi=1-\frac{f_{\star} \eta}{A_{f}(R)}$.

Claim. We have

$$
\|\varphi\|_{\infty}+\operatorname{Lip} \varphi \leq C(n, L)(D+1) / R,
$$

where $c(n, L)$ is a constant depending only on $n$ and $L$.

Proof of Claim. We observe first that

$$
|\varphi| \leq\left|1-\frac{f_{\# \chi_{R}}}{A_{f}(R)}\right|+\frac{f_{\# \chi_{R}}-f_{\#} \eta}{A_{f}(R)} \leq \frac{c D}{R}+\frac{f_{\# \chi_{R}}-f_{\#} \chi_{R-1}}{A_{f}(R)},
$$

and

$$
\operatorname{Lip} \varphi=\frac{\operatorname{Lip}\left(f_{\#} \eta\right)}{A_{f}(R)} \leq L \frac{f_{\#}\left(\chi_{R}-\chi_{R-1}\right)}{A_{f}(R)} .
$$

Thus it suffices to estimate $f_{\#}\left(\chi_{R}-\chi_{R-1}\right) / A_{f}(R)$. By Theorem 5.2 we have that

$$
\begin{aligned}
\frac{f_{\star}\left(\chi_{R}-\chi_{R-1}\right)}{A_{f}(R)} & \leq 1+\frac{c D}{R}-\frac{A_{f}(R-1)}{A_{f}(R)}\left(1-\frac{c D}{R-1}\right) \leq \frac{A_{f}(R)-A_{f}(R-1)}{A_{f}(R)}+\frac{2 c D}{R} \\
& \leq \frac{n L^{n} R^{n-1}}{L^{-n} R^{n}}+\frac{2 c D}{R}=\frac{n L^{2 n}+c D}{R},
\end{aligned}
$$

for $R>C D$.

By Lemmas 2.1 and 4.1, we have

$$
\begin{aligned}
\mathcal{F}(T) & =\mathcal{F}\left(T \left\lfloor\left(f_{\#} \eta / A_{f}(R)\right)+T\lfloor\varphi) \leq \frac{1}{A_{f}(R)} \mathcal{F}\left(T\left\lfloor f_{\#} \eta\right)+\mathcal{F}(T\lfloor\varphi)\right.\right.\right. \\
& \leq \frac{L^{k+1}\left(\|\eta\|_{\infty}+\operatorname{Lip} \eta\right)}{A_{f}(R)} \mathcal{F}\left(f^{\star} T\right)+\left(\|\varphi\|_{\infty}+\operatorname{Lip} \varphi\right) \mathcal{F}(T) \\
& \leq \frac{2 L^{k+1}}{A_{f}(R)} \mathcal{F}\left(f^{\star} T\right)+\left(\|\varphi\|_{\infty}+\operatorname{Lip} \varphi\right) \mathcal{F}(T) .
\end{aligned}
$$


If $R>2 C(n, L)(D+1)$, then (5.6) yields the estimate

$$
\mathcal{F}(T) \leq \frac{c(n, L, k)}{A_{f}(R)} \mathcal{F}\left(f^{\star} T\right)+\frac{1}{2} \mathcal{F}(T),
$$

from which the remaining inequality readily follows.

\section{Homology of normal metric currents}

In this section we assume that $X$ is a compact oriented cohomology manifold and, in addition, that $X$ is locally Lipschitz contractible. Recall that $X$ is locally Lipschitz contractible if every neighborhood $U$ of every point $x \in X$ contains a neighborhood $V \subset U$ of $x$ so that there is a Lipschitz map

$$
h:[0,1] \times V \rightarrow U
$$

so that $h_{1}(y)=y$ for every $y \in V$ and $h_{0}$ is constant. We remark that this is similar to the notion of $y$-Lipschitz contractibility in [30, Section 3.2]. For compact spaces it is not difficult to see that the two notions coincide in the sense that a locally Lipschitz contractible is $y$-Lipschitz contractible for some $y$, and a $y$-Lipschitz contractible space is locally Lipschitz contractible.

\subsection{Current homology and oriented cohomology manifolds}

The boundary map

$$
\partial_{k}: N_{k}(X) \rightarrow N_{k-1}(X)
$$

satisfies $\partial_{k-1} \partial_{k}=0$, which can be readily seen from the definition of metric currents; see also [17, Section 3]. Thus the boundary map induces a chain complex

$$
\cdots \stackrel{\partial}{\longrightarrow} N_{k}(X) \stackrel{\partial}{\longrightarrow} N_{k-1}(X) \stackrel{\partial}{\longrightarrow} \cdots \stackrel{\partial}{\longrightarrow} N_{0}(X) \longrightarrow 0
$$

As is customary we omit the subscripts from $\partial$.

We study the homology of the chain complex (6.1) for a BLD-elliptic oriented cohomology manifold $X$ and we denote the homology groups of (6.1) by

$$
H_{k}(X):=\operatorname{ker} \partial_{k} / \operatorname{im} \partial_{k+1},
$$

for $k \geq 0$.

It is known that $H_{*}(\cdot)$ defines a homology theory satisfying the Eilenberg-Steenrod axioms; see [21] and also [30] for integral currents, and [8] for the homology of normal chains and cohomology of charges. For us, homology always refers to the homology (6.2) of (6.1).

Remark 6.1. In what follows we compare the homology $H_{\star}(X)$ with the singular homology $H_{\star}^{S}(X ; \mathbb{R})$ with real coefficients. The assumption of local Lipschitz contractibility ensures that, on spaces that have the homotopy type of a CW-complex, the normal current homology $H_{\star}(X)$ coincides with singular homology $H_{\star}^{S}(X ; \mathbb{R})$; see [21, Corollary 1.6].

\subsection{Filling inequalities}

We say that a locally compact metric space $X$ admits a filling inequality for $N_{k}(X)$ if there is a constant $C>0$ such that each $T \in N_{k+1}(X)$ satisfies

$$
\text { FillVol }(\partial T) \leq C M(\partial T) .
$$


Recall that the filling volume of a current $A \in N_{k}(X)$ is defined to be

$$
\operatorname{FillVol}(A)=\inf \{M(B): \partial B=A\},
$$

the infimum over the empty set being understood as infinity. This means in particular that, if $S \in N_{k}(X)$ and $S=\partial T^{\prime}$ for some $T^{\prime} \in N_{k+1}(X)$, then there exists $T \in N_{k+1}(X)$ satisfying $S=\partial T$ and

$$
M(T) \leq C M(S) .
$$

There is a related notion of cone type inequalities introduced by Wenger [29]. A space $X$ is said to support cone type inequalities for $N_{k}(X)$ if there exists a constant $C>0$ with the property that, if $S \in \operatorname{ker} \partial_{k}$, then there exists $T \in N_{k+1}(X)$ satisfying $\partial T=S$ and

$$
M(T) \leq C \operatorname{diam}(\operatorname{spt} S) M(S) .
$$

A space $X$ supporting a cone type inequality for $N_{k}(X)$ necessarily has trivial current homology $H_{k}(X)$, whereas spaces admitting filling inequalities only require (6.3) for currents $S$ a priori known to have a filling.

Remark 6.2. In [8], De Pauw, Hardt, and Pfeffer introduce the notion of locally acyclic spaces, see [8, Definition 16.10]. Locally Lipschitz contractible spaces are locally acyclic spaces, but the connection between filling inequalities and local acyclicity is not clear to us.

In this subsection we prove that compact BLD-elliptic spaces as in Theorem 1.5 support filling inequalities.

Proposition 6.3. Let $f: \mathbb{R}^{n} \rightarrow X$ be an L-BLD map into a compact, geodesic, oriented and locally Lipschitz contractible cohomology $n$-manifold $X$, and let $0 \leq k \leq n$. Then there exists a constant $C>0$ having the property that, for every $T \in \operatorname{im} \partial_{k+1}$ there exists $S \in N_{k+1}(X)$ satisfying $\partial S=T$ and

$$
M(S) \leq C M(T) .
$$

Filling inequalities are equivalent to the closedness of the range of $\partial$. We show this using finite dimensionality of the homology.

Lemma 6.4. Let $X$ be a compact locally Lipschitz contractible metric space of finite covering dimension. Then the current homology $H_{k}(X)$ is finite dimensional for all $k \in \mathbb{N}$.

Proof. By [14, Theorem V.7.1] the space $X$ is an Euclidean neighborhood retract and by [11, Corollary A.8] it has the homotopy type of a finite CW-complex. By [21, Corollary 1.6] the normal current homology groups are isomorphic to the singular homology groups (with real coefficients), and thus finite dimensional.

Lemma 6.4 immediately yields the desired finite dimensionality as a corollary.

Corollary 6.5. Let $X$ be a compact, locally geodesic, orientable, and locally Lipschitz contractible cohomology $n$-manifold. Then the normal current homology groups $H_{k}(X)$ are finite dimensional for all $k \in \mathbb{N}$.

Lemma 6.6. Let $X$ be a compact, locally geodesic, orientable, and locally Lipschitz contractible cohomology $n$-manifold, and $k \geq 1$. Then the boundary operator

$$
\partial=\partial_{k}: N_{k}(X) \rightarrow N_{k-1}(X)
$$

has closed range.

Proof. Since im $\partial \subset \operatorname{ker} \partial_{k-1}$, we may consider $\partial$ as an operator

$$
\partial: N_{k}(X) \rightarrow \operatorname{ker} \partial_{k-1} \text {. }
$$

By Corollary 6.5 the subspace im $\partial$ has finite co-dimension in ker $\partial_{k-1}$. Then im $\partial$ is closed in ker $\partial_{k-1}$ and thus in $N_{k-1}(X)$; see e.g. [2, Corollary 2.17]. 
We are now ready for the proof of the filling inequality.

Proof of Proposition 6.3. Let $k \geq 0$ and consider the operator $\partial=\partial_{k+1}$. By Lemma 6.6, $(\operatorname{im} \partial, N)$ is a Banach space. The canonical operator

$$
\bar{\partial}: N_{k+1}(X) / \operatorname{ker} \partial \rightarrow \operatorname{im} \partial
$$

is injective and onto. By the open mapping theorem, there is a constant $0<c<\infty$ for which

$$
M(\partial T)=N(\bar{\partial}[T]) \geq c\|[T]\|_{N_{k+1}(X) / \operatorname{ker} \partial}=c \inf \{N(T-A): \partial A=0\}
$$

for every $T \in N_{k}(X)$. Let $A \in \operatorname{ker}_{k+1} \partial$. Then

$$
\text { FillVol }(\partial T) \leq M(T-A) \leq N(T-A) .
$$

This implies

$$
\text { FillVol }(\partial T) \leq c^{-1} M(\partial T),
$$

and consequently the filling inequality for $N_{k}(X)$.

\subsection{Homological boundedness}

We use the filling inequality to establish the existence of mass minimal elements in homology classes of $H_{*}(X)$.

Lemma 6.7. Let $X$ be compact, geodesic and locally Lipschitz contractible oriented cohomology manifold, and let $k \geq 0$ be an integer. Then each homology class $[S]=S+\operatorname{Im\partial } \in H_{k}(X)$ contains an element $T \in[S]$ minimizing the flat norm $\mathcal{F}$ in $[S]$. Moreover, $T$ satisfies

$$
\mathcal{F}(T)=M(T)
$$

and minimizes $\mathcal{F}$ in $[S]$ as well.

Proof. Suppose $S_{m}=S+\partial A_{m}$ is a minimizing sequence in $[S]$, and denote $B=\sup _{m} M\left(S_{m}\right)<\infty$. By Proposition 6.3, we may assume that

$$
M\left(A_{m}\right) \leq C M\left(\partial A_{m}\right)=C M\left(S-S_{m}\right) \leq C(M(S)+B)
$$

for each $m \in \mathbb{N}$, where $C$ is the constant in the claim of Proposition 6.3. Thus

$$
\sup _{m} N\left(A_{m}\right)<\infty .
$$

By passing to a subsequence we may assume that the sequence $\left(A_{m}\right)$ converges weakly to a normal current $A \in N_{k+1}(X)$. By the lower semicontinuity of the mass,

$$
M(S+\partial A) \leq \liminf _{m} M\left(S+\partial A_{m}\right)=\inf \{M(T): T \in[A]\} .
$$

Thus $S+\partial A$ is a mass minimizer in $[S]$.

Let $T \in[S]$ be a mass minimizer in $[S]$. Then the inequality $\mathcal{F}(T) \leq M(T)$ holds automatically. Further, for any $A \in N_{k+1}(X)$,

$$
M(T) \leq M(T-\partial A) \leq M(T-\partial A)+M(A) .
$$

Thus, taking infimum over $A \in N_{k+1}(X)$ yields $M(T) \leq \mathcal{F}(T)$.

The equality $\mathcal{F}(T)=M(T)$ implies that, for any $A \in S+\operatorname{im} \partial$ and $B \in N_{k+1}(X)$,

$$
\mathcal{F}(T)=M(T) \leq M(A-\partial B) \leq M(A-\partial B)+M(B) .
$$

Taking infimum over $B$ proves the last claim. 


\section{Proof of a non-smooth Bonk-Heinonen theorem}

To prove Theorem 1.5 we introduce a norm $|\cdot|: H_{k}(X) \rightarrow[0, \infty)$ on the homology group $H_{k}(X)$ by

$$
c \mapsto \inf \{M(T): T \in C\} .
$$

By Lemma 6.7 each homology class $c \in H_{k}(X)$ contains an element of minimal norm, and in particular $|c|>0$ if and only if $c \neq 0$.

Proof of Theorem 1.5. By scaling the map and the metric of the space $X$ we may assume $D=\operatorname{diam} X=1$. Let $m=\operatorname{dim} H_{k}(X)$. Then there exists linearly independent homology classes $\left[T_{1}\right], \ldots\left[T_{m}\right] \in H_{k}(X)$ satisfying

$$
\left|\left[T_{i}\right]\right|=M\left(T_{i}\right)=1 \text { and }\left|\left[T_{i}\right]-\left[T_{j}\right]\right| \geq 1 / 2 \text { if } i \neq j,
$$

for $i, j \in\{1, \ldots, m\}$. Let $S_{i j}$ be mass minimizers in the homology class $\left[T_{i}-T_{j}\right]$. By Lemma 6.7, we have

$$
1 / 2 \leq\left|\left[T_{i}-T_{j}\right]\right|=M\left(S_{i j}\right)=\mathscr{F}\left(S_{i j}\right) \leq \mathcal{F}\left(T_{i}-T_{j}\right)
$$

for $i \neq j$. Let $R=2 C$, where $C$ is the constant in Theorem 5.2. By Theorem 5.4, there are constants $a=a(n, L)>$ 0 and $b=b(n, L)>0$, depending only on $n$ and $L$, for which

$$
\mathcal{F}_{B(R)}\left(f^{\star} T_{i}-f^{\star} T_{j}\right) \gtrsim_{n, L} A_{f}(R) \mathcal{F}\left(T_{i}-T_{j}\right) \geq a(n, L) \frac{R^{n}}{|X|}
$$

and

$$
\left\|f^{\star} T_{i}\right\|\left(B_{R}\right) \simeq_{n, L} A_{f}(R) \geq b(n, L) \frac{R^{n}}{|X|}
$$

for all $i, j \in\{1, \ldots, m\}, i \neq j$.

Let $\eta: \mathbb{R}^{n} \rightarrow \mathbb{R}$ be the Lipschitz function $x \mapsto(1-\operatorname{dist}(B(R), x))_{+}$, and define $S_{i}:=|X|\left(f^{\star} T_{i}\right)\lfloor\eta$ for each $i=1, \ldots, m$. Note that spt $\eta \subset B(R+1)=B(2 C+1)$. Thus $S_{i}$ is supported in $B(2 C+1)$ for each $i=1, \ldots, m$. By (7.2)

$$
M\left(S_{i}\right)=N\left(S_{i}\right) \leq b(2 C+1)^{n}
$$

for each $i=1, \ldots, m$. Moreover, by (7.1) we have

$$
\mathcal{F}_{B(2 C+1)}\left(S_{i}-S_{j}\right) \geq \mathcal{F}_{B(2 C)}\left(S_{i}-S_{j}\right) \geq a(2 C)^{n}
$$

whenever $i \neq j$; cf Lemma 2.1.

Thus

$$
\left\{S_{1}, \ldots, S_{m}\right\} \subset N\left(\bar{B}(2 C+1), b(2 C+1)^{n}\right),
$$

where, for an compact set $K \subset \mathbb{R}^{n}$, and $\lambda \geq 0$,

$$
N(K, \lambda)=\left\{T \in N_{k}\left(\mathbb{R}^{n}\right): \text { spt } T \subset U, N(T) \leq \lambda\right\} .
$$

By Theorem 2.2 the right-hand side in (7.3) is compact in $\mathcal{F}_{B(2 C+1)}$. Therefore there is an upper bound $m(n, L)$, depending only on $n$ and $L$, on the cardinality of a finite set $S$ in $N\left(B(2 C+1), b(2 C+1)^{n}\right)$ having the property that

$$
\mathcal{F}_{B(2 C+1)}\left(S-S^{\prime}\right) \geq b(2 C)^{n}
$$

whenever $S, S^{\prime} \in \mathcal{S}$ and $S \neq S^{\prime}$. We conclude that $m=\operatorname{dim} H_{k}(X) \leq m(n, L)$. The proof is complete. 


\section{A Local Euclidean bilipschitz embeddability of BLD-elliptic spaces}

In this appendix we prove the following embeddability theorem mentioned in the introduction.

Theorem A.1. Let $X$ be a locally geodesic, orientable cohomology manifold admitting a BLD-map $f: \mathbb{R}^{n} \rightarrow X$. Let $x \in X$. For every radius $r>0$, for which there exists $y \in f^{-1}(x)$ such that $U(y, r)$ is a normal neighborhood of $y, B_{r}(x)$ is bilipschitz equivalent to a subset of a Euclidean space.

In the proof we use Almgren's theory of $Q$-valued maps. We refer to [7] for a recent exposition. Denote by $\mathcal{A}_{Q}\left(\mathbb{R}^{n}\right)$ the space of unordered $Q$-tuples of points in $\mathbb{R}^{n}$. For the purpose of introducing a metric, we formally define

$$
\mathcal{A}_{Q}\left(\mathbb{R}^{n}\right)=\left\{\sum_{i=1}^{Q} \delta_{x_{i}}: x_{1}, \ldots, x_{Q} \in \mathbb{R}^{n}\right\},
$$

where $\delta_{x}$ is the Dirac mass at $x \in \mathbb{R}^{n}$. Given $T_{1}, T_{2} \in \mathcal{A}_{Q}\left(\mathbb{R}^{n}\right)$, suppose

$$
T_{1}=\sum_{i=1}^{Q} \delta_{x_{i}}, \quad T_{2}=\sum_{i=1}^{Q} \delta_{y_{i}},
$$

and define

$$
d_{Q}\left(T_{1}, T_{2}\right)=\min \left\{\left(\sum_{i=1}^{Q}\left|x_{i}-y_{\sigma(i)}\right|^{2}\right)^{1 / 2}: \sigma \in S_{Q}\right\},
$$

where $S_{Q}$ denotes the set of permutations of $\{1, \ldots, Q\}$. A key property of $\mathcal{A}_{Q}\left(\mathbb{R}^{n}\right)$ is the following bilipschitz embedding result.

Theorem A.2. [7, Theorem 2.1] There exists $N=N(Q, n)$ and a bilipschitz map

$$
\xi: \mathcal{A}_{Q}\left(\mathbb{R}^{n}\right) \rightarrow \mathbb{R}^{N}
$$

Let $x \in X$ and $r>0$, and suppose that $y \in f^{-1}(x)$ has the property that $U=: U(y, r)$ is a normal neighborhood of $y$. Set $Q=i_{f}(y)$. We define a $Q$-valued map $g_{f}: B_{r}(x) \rightarrow \mathcal{A}_{Q}\left(\mathbb{R}^{n}\right)$ by

$$
x \mapsto \sum_{z \in f^{-1}(x) \cap U} i_{f}(z) \delta_{z} .
$$

Lemma A.3. Let $f: X \rightarrow Y$ be a BLD-map between locally geodesic, oriented cohomology manifolds. Then, the map $\mathrm{g}: B_{r}(x) \rightarrow \mathcal{A}_{Q}\left(\mathbb{R}^{n}\right)$ is a bilipschitz embedding.

Proof. Let $p, q \in B_{r}(x) \backslash f B_{f}$. By Lemma 3.3 and its proof, there is a bijection

$$
\psi: f^{-1}(p) \cap U \rightarrow f^{-1}(q) \cap U
$$

satisfying

$$
d(p, q) / L \leq d(z, \psi(z)) \leq L d(p, q)
$$

for each $x \in f^{-1}(p) \cap U$. Thus

$$
d_{Q}\left(g_{f}(p), g_{f}(q)\right) \leq\left(\sum_{z \in f^{-1}(p) \cap U} d(z, \psi(z))^{2}\right)^{1 / 2} \leq L \sqrt{Q} d(p, q) .
$$

For the opposite inequality let $f^{-1}(p) \cap U=\left\{x_{1}, \ldots, x_{Q}\right\}, f^{-1}(q) \cap U=\left\{y_{1}, \ldots, y_{Q}\right\}$, and $\sigma \in S_{Q}$. Then, for each $i=1, \ldots, Q$,

$$
\left|x_{i}-y_{\sigma(i)}\right| \geq \frac{1}{L} \ell\left(f \circ\left[x_{i}, y_{\sigma(i)}\right]\right) \geq \frac{1}{L} d(p, q),
$$


where $\left[x_{i}, y_{\sigma(i)}\right]$ denotes the geodesic line segment from $x_{i}$ to $y_{\sigma(i)}$. Thus

$$
d_{Q}\left(g_{f}(p), g_{f}(q)\right) \geq \sqrt{Q} d(p, q) / L .
$$

We have established the bilipschitz condition for points $p, q$ in the dense set $B_{r}(x) \backslash f B_{f}$, whence it follows for all $p, q \in B_{r}(x)$.

Proof of Theorem A.1. Let $x \in X$ and let $r>0$ be a radius with the property that there exists $y \in f^{-1}(x)$ for which $U=U(y, r)$ is a normal neighborhood of $y$. Set $Q=i_{f}(y)$ and consider the map $g_{f}: B_{r}(x) \rightarrow \mathcal{A}_{Q}\left(\mathbb{R}^{n}\right)$. Then the map

$$
\xi \circ g: B_{r}(x) \rightarrow \mathbb{R}^{N},
$$

where $\xi: \mathcal{A}_{Q}\left(\mathbb{R}^{n}\right) \rightarrow \mathbb{R}^{N}$ is the map of Theorem A.2, is bilipschitz.

Remark A.4. Since the index $i_{f}$ of a BLD-map $f: \mathbb{R}^{n} \rightarrow X$ is bounded by a constant depending only on $n$ and $L$, the bilipschitz constant of $\xi \circ g$ and the dimension $N$ are also bounded by constants depending only on $n$ and $L$.

Acknowledgement: P.P. was supported in part by the Academy of Finland project \#297258.

\section{References}

[1] Martina Aaltonen and Pekka Pankka. Local monodromy of branched covers and dimension of the branch set. Ann. Acad. Sci. Fenn. Math., 42(1):487-496, 2017.

[2] Y. A. Abramovich and C. D. Aliprantis. An invitation to operator theory, volume 50 of Graduate Studies in Mathematics. American Mathematical Society, Providence, RI, 2002.

[3] Frederick J. Almgren Jr. Almgren's big regularity paper, volume 1 of World Scientific Monograph Series in Mathematics. World Scientific Publishing Co., Inc., River Edge, NJ, 2000. Q-valued functions minimizing Dirichlet's integral and the regularity of area-minimizing rectifiable currents up to codimension 2, With a preface by Jean E. Taylor and Vladimir Scheffer.

[4] Luigi Ambrosio and Bernd Kirchheim. Currents in metric spaces. Acta Math., 185(1):1-80, 2000.

[5] Mario Bonk and Juha Heinonen. Quasiregular mappings and cohomology. Acta Math., 186(2):219-238, 2001.

[6] Glen E. Bredon. Sheaf theory, volume 170 of Graduate Texts in Mathematics. Springer-Verlag, New York, second edition, 1997.

[7] Camillo De Lellis and Emanuele Nunzio Spadaro. Q-valued functions revisited. Mem. Amer. Math. Soc., 211(991):vi+79, 2011.

[8] Th. De Pauw, R. M. Hardt, and W. F. Pfeffer. Homology of normal chains and cohomology of charges. Mem. Amer. Math. Soc., 247(1172): $v+115,2017$.

[9] Herbert Federer and Wendell H. Fleming. Normal and integral currents. Ann. of Math. (2), 72:458-520, 1960.

[10] Misha Gromov. Metric structures for Riemannian and non-Riemannian spaces. Modern Birkhäuser Classics. Birkhäuser Boston Inc., Boston, MA, english edition, 2007. Based on the 1981 French original, With appendices by M. Katz, P. Pansu and S. Semmes, Translated from the French by Sean Michael Bates.

[11] Allen Hatcher. Algebraic topology. Cambridge University Press, Cambridge, 2002.

[12] Juha Heinonen, Pekka Koskela, Nageswari Shanmugalingam, and Jeremy Tyson. Sobolev spaces on metric measure spaces: an approach based on upper gradients. New Mathematical Monographs. Cambridge University Press, United Kingdom, first edition, 2015.

[13] Juha Heinonen and Seppo Rickman. Geometric branched covers between generalized manifolds. Duke Math. J., 113(3):465-529, 2002.

[14] Sze-tsen Hu. Theory of retracts. Wayne State University Press, Detroit, 1965.

[15] I. Kangasniemi. Sharp cohomological bound for uniformly quasiregularly elliptic manifolds. ArXiv e-prints, November 2017.

[16] Bernd Kirchheim. Rectifiable metric spaces: local structure and regularity of the Hausdorff measure. Proc. Amer. Math. Soc., 121(1):113-123, 1994.

[17] Urs Lang. Local currents in metric spaces. J. Geom. Anal., 21(3):683-742, 2011.

[18] Rami Luisto. A characterization of BLD-mappings between metric spaces. J. Geom. Anal., 27(3):2081-2097, 2017.

[19] O. Martio and J. Väisälä. Elliptic equations and maps of bounded length distortion. Math. Ann., 282(3):423-443, 1988.

[20] P. Mattila and S. Rickman. Averages of the counting function of a quasiregular mapping. Acta Math., 143(3-4):273-305, 1979. 
[21] Ayato Mitsuishi. The coincidence of the current homology and the measure homology via a new topology on spaces of Lipschitz maps. arXiv:1403.5518 [math.AT].

[22] Jani Onninen and Kai Rajala. Quasiregular mappings to generalized manifolds. J. Anal. Math., 109:33-79, 2009.

[23] Pekka Pankka. Mappings of bounded mean distortion and cohomology. Geom. Funct. Anal., 20(1):229-242, 2010.

[24] Pekka Pankka and Elefterios Soultanis. Metric currents and Polylipschitz forms. arXiv:1902.06106 [math.MG].

[25] Eden Prywes. A Bound on the Cohomology of Quasiregularly Elliptic Manifolds. Preprint. arXiv:1806.05306 [math.DG].

[26] Seppo Rickman. Quasiregular mappings, volume 26 of Ergebnisse der Mathematik und ihrer Grenzgebiete (3) [Results in Mathematics and Related Areas (3)]. Springer-Verlag, Berlin, 1993.

[27] Jussi Väisälä. Discrete open mappings on manifolds. Ann. Acad. Sci. Fenn. Ser. A I No., 392:10, 1966.

[28] Frank W. Warner. Foundations of differentiable manifolds and Lie groups, volume 94 of Graduate Texts in Mathematics. Springer-Verlag, New York-Berlin, 1983. Corrected reprint of the 1971 edition.

[29] Stefan Wenger. Isoperimetric inequalities of Euclidean type in metric spaces. GAFA, 15(2):534-554, 2005.

[30] Stefan Wenger. Flat convergence for integral currents in metric spaces. Calc. Var. Partial Differential Equations, 28(2):139-160, 2007. 